TITLE:

\title{
Aerodynamic forces and vortical structures in flapping butterfly's forward flight
}

\section{AUTHOR(S):}

Yokoyama, Naoto; Senda, Kei; lima, Makoto; Hirai, Norio

\section{CITATION:}

Yokoyama, Naoto ... [et al]. Aerodynamic forces and vortical structures in flapping butterfly's forward flight. Physics of Fluids 2013, 25(2): 021902.

ISSUE DATE:

2013-02

URL:

http://hdl.handle.net/2433/172547

RIGHT:

(C) 2013 American Institute of Physics 


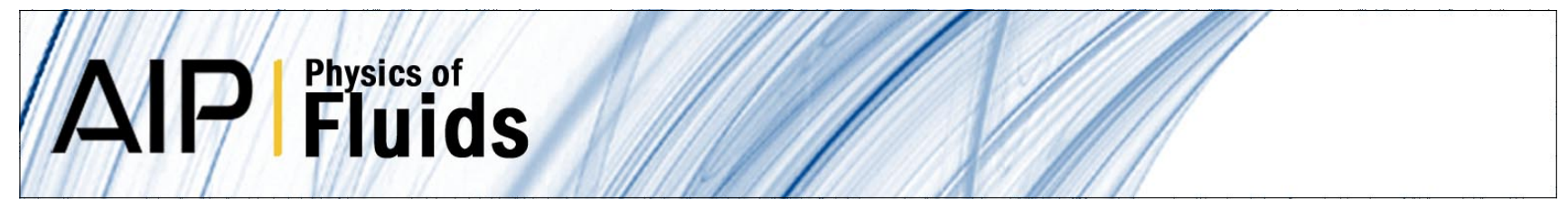

\section{Aerodynamic forces and vortical structures in flapping butterfly's forward flight}

Naoto Yokoyama, Kei Senda, Makoto lima, and Norio Hirai

Citation: Phys. Fluids 25, 021902 (2013); doi: 10.1063/1.4790882

View online: http://dx.doi.org/10.1063/1.4790882

View Table of Contents: http://pof.aip.org/resource/1/PHFLE6/v25/i2

Published by the American Institute of Physics.

\section{Related Articles}

Three-dimensional flow structure and aerodynamic loading on a revolving wing Phys. Fluids 25, 034101 (2013)

On the interaction of shock waves and sound waves in transonic buffet flow Phys. Fluids 25, 026101 (2013)

Thermochemical nonequilibrium modeling of a low-power argon arcjet wind tunnel J. Appl. Phys. 113, 053304 (2013)

Biomimetic flow control based on morphological features of living creatures Phys. Fluids 24, 121302 (2012)

Study of mechanisms and factors that influence the formation of vortical wake of a heaving airfoil Phys. Fluids 24, 103601 (2012)

\section{Additional information on Phys. Fluids}

Journal Homepage: http://pof.aip.org/

Journal Information: http://pof.aip.org/about/about_the_journal

Top downloads: http://pof.aip.org/features/most_downloaded

Information for Authors: http://pof.aip.org/authors

\section{ADVERTISEMENT}

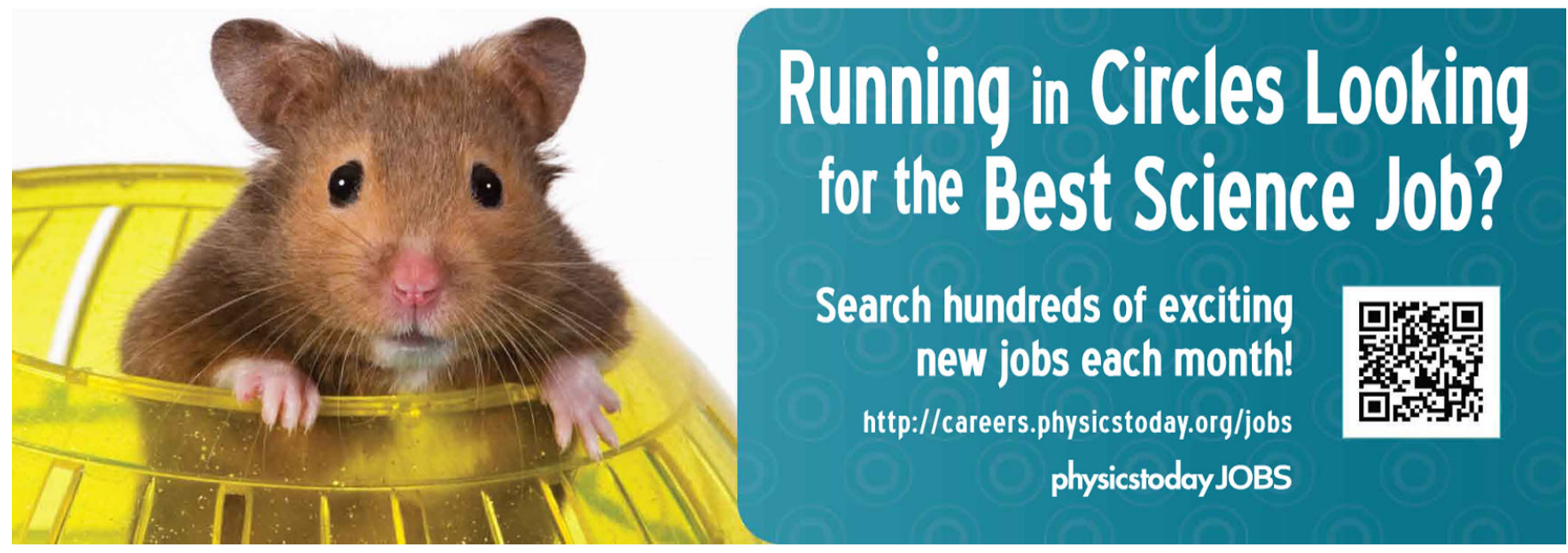


PHYSICS OF FLUIDS 25, 021902 (2013)

\title{
Aerodynamic forces and vortical structures in flapping butterfly's forward flight
}

\author{
Naoto Yokoyama, ${ }^{1, a)}$ Kei Senda, ${ }^{1}$ Makoto lima,${ }^{2}$ and Norio Hirai ${ }^{3}$ \\ ${ }^{1}$ Department of Aeronautics and Astronautics, Kyoto University, Kyoto 606-8501, Japan \\ ${ }^{2}$ Department of Mathematical and Life Sciences, Hiroshima University, \\ Higashi-Hiroshima 739-8521, Japan \\ ${ }^{3}$ Graduate School of Life and Environmental Sciences, Osaka Prefecture University, \\ Sakai 599-8531, Japan
}

(Received 27 July 2012; accepted 28 January 2013; published online 21 February 2013)

Forward flights of a bilaterally symmetrically flapping butterfly modeled as a fourlink rigid-body system consisting of a thorax, an abdomen, and left and right wings are numerically simulated. The joint motions of the butterflies are adopted from experimental observations. Three kinds of the simulations, distinguished by ways to determine the position and attitude of the thorax, are carried out: a tethered simulation, a prescribed simulation, and free-flight simulations. The upward and streamwise forces as well as the wake structures in the tethered simulation, where the thorax of the butterfly is fixed, reasonably agree with those in the corresponding tethered experiment. In the prescribed simulation, where the thoracic trajectories as well as the joint angles are given by those observed in a free-flight experiment, it is confirmed that the butterfly can produce enough forces to achieve the flapping flights. Moreover, coherent vortical structures in the wake and those on the wings are identified. The generation of the aerodynamic forces due to the vortical structures are also clarified. In the free-flight simulation, where only the joint angles are given as periodic functions of time, it is found that the free flight is longitudinally unstable because the butterfly cannot maintain the attitude in a proper range. Focusing on the abdominal mass, which largely varies owing to feeding and metabolizing, we have shown that the abdominal motion plays an important role in periodic flights. The necessity of control of the thoracic attitude for periodic flights and maneuverability is also discussed. (c) 2013 American Institute of Physics. [http://dx.doi.org/10.1063/1.4790882]

\section{INTRODUCTION}

Human-made aircraft mostly have fixed wings or rotary wings. The wings except some small parts are steady. On the other hand, most flying animals on the earth such as insects and birds flap their wings. We have much to learn from the insects which have chosen the flapping flight as a result of the natural selection since the number of the species of the insects is the largest on the earth. The aerodynamics of the insect flight are reviewed in Refs. 1-3.

Flying animals must produce aerodynamic forces enough to sustain their weight against the gravity and to overcome the drag. Moreover, the direction of the aerodynamic force should be controlled for flight maneuvering; the direction should be even in transverse to turn around. The flying animals produce the aerodynamic forces as the reactions to the force produced when the motion of their wings change the momenta of the surrounding air. The associated flow around the wings is characterized by coherent vortices, which are generated by separation from the flapping wings and interact with the wings and other vortices. Such interactions are complicated from the aerodynamic point of view, and non-trivial behaviors of flapping models have been reported:

\footnotetext{
a)Electronic mail: yokoyama@kuaero.kyoto-u.ac.jp.
} 
even up-down symmetric flaps of wings lead to unidirectional motion in both vertical and horizontal directions, ${ }^{4,5}$ depending on the model details. Bilateral asymmetry of flaps is experimentally found in in-flight turns of fruit flies. ${ }^{6}$ The maneuverability of the flapping-flight animals resulting from the variability of their flapping motions is necessary to survive.

The characteristics of the flow, which determines the forces and the torques acting on the wings, are often categorized by vortical patterns in the wake of flying animals. Doughnut-shaped vortex rings are often shed by slowly-traveling animals' wings into the wake at the transitions from downstrokes to upstrokes while undulating cylindrical vortices trailing behind each wing tip are often found in the wakes of fast traveling animals. ${ }^{7}$ The wake structures created by forward-flight or hovering flapping "flyers", such as a hummingbird, a bat, and a foil, have experimentally or numerically been visualized. ${ }^{8-10}$ A ladder-like vortical structure in the wake of a flapping butterfly was proposed. ${ }^{11}$ Flow structures produced by hovering insects have also been visualized. ${ }^{12,13}$ The relation between vortical structures induced by the flap motions and aerodynamic forces of a tethered fruit fly was experimentally investigated. ${ }^{14}$ The leading-edge vortex created by dynamic stall enhances lift of hawkmoth's hovering flight. ${ }^{15}$ The importance of reattachment of the leading-edge vortices in lift generation of a butterfly was also reported. ${ }^{16}$ The forces due to vortex rings in the wakes were theoretically estimated. ${ }^{17}$ The forces and torques acting on insects' flapping wings have been estimated numerically. ${ }^{12,13,18-20}$

The flights of butterflies and moths appear to be energy-efficient, since they are known to be able to fly with smaller flapping frequencies than other insects comparable in size to them (see Ref. 21 and references therein). We remark that, when compared with the fixed wing, the energy efficiency of flapping flight was shown to be better in a two-dimensional model, ${ }^{22}$ even though the flapping motion appears to require additional energy consumption. Moreover, the selection rule of a flapping frequency was discussed, ${ }^{23}$ and it was concluded that the optimal frequency of a flapping foil in two-dimensional flow is the frequency of the advectively most unstable mode of the wakes. ${ }^{24}$ The advantage of the flapping forward flights in three-dimensional flow has not been investigated in detail. The mechanism of butterflies' flights with small flapping frequencies needs to be clarified.

Natural flyers obtain information of their attitudes and locations by their sensory organs..$^{25,26}$ According to the information, the flyers control their attitudes and locations by changing their flapping motions explicitly. Cabbage white butterfly alters the angle of the joint between the thorax and the abdomen to stabilize their thoracic pitching angles (see Ref. 27 and reference therein). Hawkmoths maintain their positions and attitudes during hovering flights with redundantly huge number of degrees of freedom as an inverse problem. ${ }^{28}$ The mechanisms of the control are attracting the interests of researchers also in engineering. ${ }^{29}$ Stabilization in butterflies' flights due to elastic deformation of wings was also reported. ${ }^{30}$ We will see the necessity of the control from the viewpoint of the fluid dynamics.

Among these previous studies, we study the flapping flights of relatively large-size butterflies. Turbulent flows made by the flapping flights and the abdominal motion are the primary distinctions from the flapping flights of other insects. In this study, bilaterally symmetrically flapping butterflies in forward flights are numerically simulated to clarify the relation between aerodynamic forces and flow structures characterized by vortices. Three kinds of the simulations, distinguished by ways to determine the position and the attitude of the thorax of the butterfly, are carried out: a tethered simulation, a prescribed simulation, and free-flight simulations. In all the simulations joint motions of the butterflies are adopted from experiments with living butterflies. In the tethered simulation, where the thorax of the butterfly is fixed, the forces caused by the flaps are compared with the corresponding experiment. The wake structures visualized by passive tracers in the simulation are also compared with those visualized by smoke in the experiment. The aerodynamic forces and torque during a free flight are numerically obtained in the prescribed simulation, where the thoracic trajectories as well as the joint angles are given by those observed in the free-flight experiment. The torques and powers generated by the joints are confirmed to be consistent with the biological facts. Vortical structures are also visualized and forces due to the vortical structures are identified. In the free-flight simulations, where only the joint angles are given and the thoracic trajectories are numerically obtained, feasibility of the free flights are discussed. 


\section{NUMERICAL SCHEME}

\section{A. Flow field}

The governing equation of the incompressible flow is the Navier-Stokes equation:

$$
\begin{aligned}
\frac{\partial \boldsymbol{u}}{\partial t}+(\boldsymbol{u} \cdot \nabla) \boldsymbol{u} & =-\frac{\nabla p}{\rho_{\mathrm{air}}}+v \nabla^{2} \boldsymbol{u}+\boldsymbol{f}_{\mathrm{IB}}, \\
\nabla \cdot \boldsymbol{u} & =0,
\end{aligned}
$$

where $\boldsymbol{u}$ is the velocity of the flow, and $p$ is the pressure. The interactions between the flow and the butterfly are calculated by the immersed boundary method. ${ }^{31}$ The interactions are introduced as the boundary force $f_{\mathrm{IB}}$. The values of the air properties are adopted from those at $25^{\circ} \mathrm{C}$ : the density $\rho_{\text {air }}=1.184 \mathrm{~kg} / \mathrm{m}^{3}$ and the kinematic viscosity $v_{\text {air }}=1.54 \times 10^{-5} \mathrm{~m}^{2} / \mathrm{s}$. However, in this study, the values of the kinematic viscosity, $v=2 v_{\text {air }}$ or $3 v_{\text {air }}$, larger than the actual value are also adopted to avoid numerical oscillation. The forces and torques acting on the butterfly do not depend much on the kinematic viscosity in this range. The dependence on the kinematic viscosity as well as other numerical parameters is checked in the Appendix.

The direction of the main stream is taken as the positive $x$ direction, the vertically upward direction as the positive $y$ direction, and the horizontal direction as the $z$ direction. The directions are expressed by the Cartesian unit vectors $\boldsymbol{e}_{x}, \boldsymbol{e}_{y}$, and $\boldsymbol{e}_{z}$ in Fig. 1. The boundary conditions of the flows are as follows. The velocity is constant on the inflow boundary plane: $\boldsymbol{u}=u_{0} \boldsymbol{e}_{x}$. The Sommerfeld condition is employed as the outflow condition. The boundary condition of the pressure is the Neumann boundary condition $\partial p / \partial x=0$ on both inflow and outflow plane. The streamwise length of the computational domain is $L_{x}=5 \times 10^{-1} \mathrm{~m}$, which is approximately 18 times as long as the mean wing-chord length. The periodic boundary condition is employed for the transverse boundaries. The transverse length of the computational domain is $L_{y}=L_{z}=2.5 \times 10^{-1} \mathrm{~m}$, which

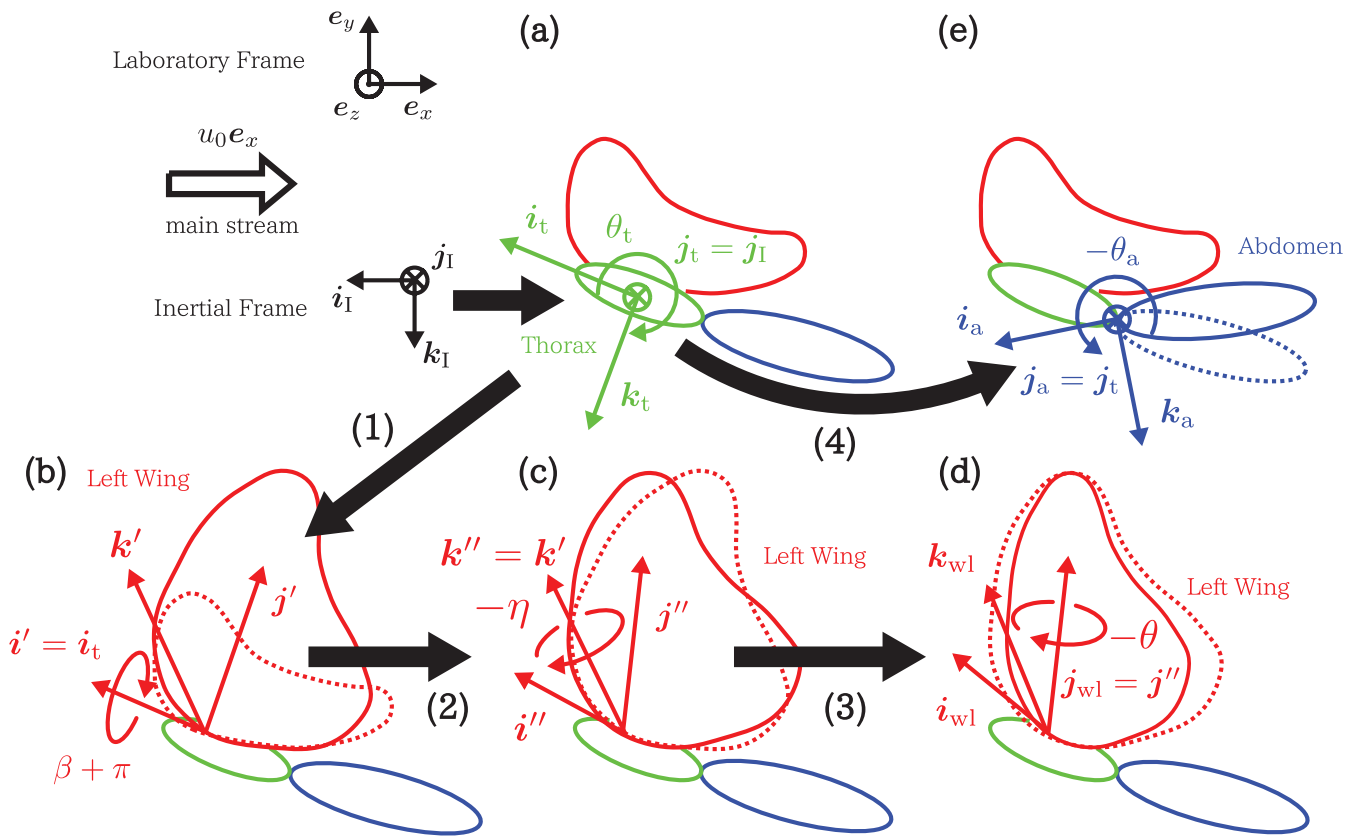

FIG. 1. Definitions of coordinates and the Euler angles. The positive directions of the thoracic and joint angles $\theta_{\mathrm{t}}, \theta_{\mathrm{a}}, \beta, \eta$, and $\theta$ are expressed by the round arrows. (a) First, the coordinate of the left wing corresponds to that of the thorax on the right side. (d) After the three rotations (1: about the $\boldsymbol{i}_{\mathrm{t}}$ axis by $\beta+\pi, 2$ : about the $\boldsymbol{k}^{\prime}$ axis by $-\eta, 3$ : about the $\boldsymbol{j}^{\prime \prime}$ axis by $-\theta$ ) the coordinate corresponds to $\left(\boldsymbol{i}_{\mathrm{wl}}, \boldsymbol{j}_{\mathrm{wl}}, \boldsymbol{k}_{\mathrm{wl}}\right)$. (e) The coordinate of the thorax corresponds to that of the abdomen after the rotation about the $\boldsymbol{j}_{\mathrm{t}}$ axis by $-\theta_{\mathrm{a}}$ (4). The positive directions of the Euler angles of the rotations $\phi_{i j}$ are determined by the right-hand rule. 
is approximately 5 times as long as the wing-tip length. The results are not affected by the values of $L_{x}, L_{y}$, and $L_{z}$ (see the Appendix).

The Navier-Stokes equation is spatially discretized by Eulerian uniform grids in the Cartesian coordinate. The spatial resolution is $N_{x} \times N_{y} \times N_{z}=4096 \times 512 \times 512$. The fourth-order central difference is used for the derivatives in the $x$ direction. The second-order forward and backward differences also used near the inflow and outflow boundaries, respectively. The derivatives in the $y$ and $z$ directions are evaluated with the Fourier transforms. The Poisson equation with pentadiagonal matrix is solved by the Gaussian elimination thanks to the periodic boundary conditions in the $y$ and $z$ directions.

The time integration of the flow is made by the fractional step method from $t_{n}$ to $t_{n+1}=t_{n}$ $+\Delta t$, where $\Delta t$ is a time increment, as follows:

1. The fractional step of the advective term and the viscous term, $\boldsymbol{H}=-(\boldsymbol{u} \cdot \nabla) \boldsymbol{u}+v \nabla^{2} \boldsymbol{u}$, is made as $\hat{\boldsymbol{u}}\left(\boldsymbol{x}, t_{n+1 / 2}\right)=\boldsymbol{u}\left(t_{n}\right)+\boldsymbol{H}\left(t_{n}\right) \Delta t$ by the second-order Runge-Kutta method. The advective term is obtained by the spectral method with de-aliasing by the $3 / 2$ method. The viscous term is implicitly added.

2. The force per unit mass at an Eulerian grid point $\boldsymbol{x}$ in the flow field is obtained according to the immersed boundary method. The velocity of the flow is interpolated on the immersed boundaries, i.e., at a Lagrangian point $\boldsymbol{X}_{n}=\boldsymbol{X}\left(t_{n}\right)$ on the surface of the butterfly as $\breve{\boldsymbol{u}}\left(\boldsymbol{X}_{n}, t_{n+1 / 2}\right)=\sum_{\boldsymbol{x}} \hat{\boldsymbol{u}}\left(\boldsymbol{x}, t_{n+1 / 2}\right) \delta_{\Delta \boldsymbol{x}}\left(\boldsymbol{x}-\boldsymbol{X}_{n}\right) \Delta \boldsymbol{x}$, where $\Delta \boldsymbol{x}=\Delta x \Delta y \Delta z$ is the volume of the Eulerian cell. The discrete $\delta$ function $\delta_{\Delta x}(\boldsymbol{x}-\boldsymbol{X})=(1 / \Delta \boldsymbol{x}) \delta_{\mathrm{s} 4}(|x-X| / \Delta x) \delta_{\mathrm{s} 4}(\mid y$ $-Y \mid / \Delta y) \delta_{\mathrm{s} 4}(|z-Z| / \Delta z)$ is given by the smoothed four-point piecewise function $\delta_{\mathrm{s} 4}$ proposed in Ref. 32. The force per unit area at $\boldsymbol{X}$ on the immersed boundaries due to the flow is given by the difference between the interpolated velocity and the velocity of the immersed boundary $\boldsymbol{U}$ as $\boldsymbol{F}\left(\boldsymbol{X}_{n}, t_{n+1 / 2}\right)=\rho_{\text {air }}\left(\check{\boldsymbol{u}}\left(\boldsymbol{X}_{n}, t_{n+1 / 2}\right)-\boldsymbol{U}_{n}\right) \Delta \boldsymbol{x} /(\Delta t \Delta \boldsymbol{X})$. Here, $\Delta \boldsymbol{X}$ is the area of the cell on the immersed boundary. The force per unit mass is obtained as $\boldsymbol{f}_{\mathrm{IB}}\left(\boldsymbol{x}, t_{n+1 / 2}\right)=-\left(1 / \rho_{\mathrm{air}}\right) \sum_{\boldsymbol{X}} \boldsymbol{F}\left(\boldsymbol{X}_{n}, t_{n+1 / 2}\right) \delta_{\Delta \boldsymbol{x}}\left(\boldsymbol{x}-\boldsymbol{X}_{n}\right) \Delta \boldsymbol{X}$. Then, the fractional step of the immersed boundary is made as $\tilde{\boldsymbol{u}}\left(t_{n+1 / 2}\right)=\hat{\boldsymbol{u}}\left(t_{n+1 / 2}\right)+\boldsymbol{f}_{\mathrm{IB}}\left(t_{n+1 / 2}\right) \Delta t$.

3. The pressure term is calculated. The pressure function $P=p / \rho_{\text {air }}$ is obtained by solving the Poisson equation $\nabla^{2} P=\nabla \cdot \tilde{\boldsymbol{u}}\left(t_{n+1 / 2}\right) / \Delta t$. The fractional step of the pressure is made as $\boldsymbol{u}\left(t_{n+1}\right)=\tilde{\boldsymbol{u}}\left(t_{n+1 / 2}\right)-\nabla P \Delta t$, and this is the velocity of the flow at $t_{n+1}$.

We adopt $T / 4000$ or $T / 8000$ as the time increment $\Delta t$ in this study, where $T$ is a flapping period.

\section{B. Butterfly}

A chestnut tiger butterfly (Parantica sita) is modeled as four rigid bodies: a spheroidal thorax, a spheroidal abdomen, and left and right thin flat wings. The functional form of the shape of the wings are given by the polynomial approximation of the average shape of the wings of living butterflies. Although the butterfly has a forewing and a hindwing on one side, the forewing and hindwing are modeled as a unified wing. The thorax and the abdomen, the thorax and the left wing, and the thorax and the right wing are connected with joints with three degrees of freedom (DOF). We have used the parameters of two individual butterflies as the dimensions of the modeled butterfly. The dimensions are summarized in Table I.

The relations between the thoracic mass and the thoracic size, and between the masses of the wings and the wing-tip length are statistically obtained by measuring the masses and the lengths of the disassembled parts. Then, the masses of the thorax and the wings are, respectively, estimated by measuring the thoracic size and the wing-tip length of the living butterfly. Because the total mass of the living butterfly can be measured directly, the masses of the thorax and the wings subtracted from the total mass give the abdominal mass. Note that the actual values of the abdominal mass largely vary during the experiments.

The state of the butterfly is given by $\boldsymbol{\theta}=\left(\boldsymbol{x}_{\mathrm{t}}, \boldsymbol{\phi}_{\mathrm{t}}, \boldsymbol{\phi}_{\mathrm{a}}, \boldsymbol{\phi}_{\mathrm{wl}}, \boldsymbol{\phi}_{\mathrm{wr}}\right) \in \mathbb{R}^{15}$ and its time derivative $\dot{\boldsymbol{\theta}}$, where $\boldsymbol{x}_{\mathrm{t}}$ is the position of the center of mass (COM) of the thorax, and $\boldsymbol{\phi}_{\mathrm{t}}$ represents the attitude of the thorax. The joint angles of the abdomen, the left wing, and the right wing are denoted by $\boldsymbol{\phi}_{\mathrm{a}}, \boldsymbol{\phi}_{\mathrm{wl}}$, and $\boldsymbol{\phi}_{\mathrm{wr}}$, respectively. Note that each of $\boldsymbol{x}_{\mathrm{t}}, \boldsymbol{\phi}_{\mathrm{t}}, \boldsymbol{\phi}_{\mathrm{a}}, \boldsymbol{\phi}_{\mathrm{wl}}$, and $\boldsymbol{\phi}_{\mathrm{wr}}$ has three components. The 
TABLE I. Physical properties of butterflies (chestnut tiger)

\begin{tabular}{lcc}
\hline \hline Parameters & Individual A & Individual B \\
\hline Total mass $m(\mathrm{~kg})$ & $3.38 \times 10^{-4}$ & $3.36 \times 10^{-4}$ \\
Wing loading $m g /\left(2 S_{\mathrm{w}}\right)\left(\mathrm{N} / \mathrm{m}^{2}\right)$ & 1.13 & 1.32 \\
Thoracic mass $m_{\mathrm{t}}(\mathrm{kg})$ & $1.20 \times 10^{-4}$ & $8.28 \times 10^{-5}$ \\
Thoracic length $2 l_{\mathrm{t}}(\mathrm{m})$ & $1.24 \times 10^{-2}$ & $1.05 \times 10^{-2}$ \\
Thoracic width $2 w_{\mathrm{t}}(\mathrm{m})$ & $5.40 \times 10^{-3}$ & $5.50 \times 10^{-3}$ \\
Abdominal mass $m_{\mathrm{a}}(\mathrm{kg})$ & $1.68 \times 10^{-4}$ & $2.11 \times 10^{-4}$ \\
Abdominal length $2 l_{\mathrm{a}}(\mathrm{m})$ & $2.14 \times 10^{-2}$ & $2.10 \times 10^{-2}$ \\
Abdominal width $2 w_{\mathrm{a}}(\mathrm{m})$ & $5.70 \times 10^{-3}$ & $4.55 \times 10^{-3}$ \\
Wing mass $m_{\mathrm{w}}(\mathrm{kg})$ & $2.52 \times 10^{-5}$ & $2.10 \times 10^{-5}$ \\
Wing-tip length $y_{\text {tip }}(\mathrm{m})$ & $5.47 \times 10^{-2}$ & $5.03 \times 10^{-2}$ \\
Wing area $S_{\mathrm{w}}(\mathrm{m})$ & $1.47 \times 10^{-3}$ & $1.24 \times 10^{-3}$ \\
\hline \hline
\end{tabular}

components of $\boldsymbol{\theta}$ are divided into the thoracic coordinates denoted by $\boldsymbol{\theta}_{1}$ and the joint coordinates by $\boldsymbol{\theta}_{2} ; \boldsymbol{\theta}=\left(\boldsymbol{\theta}_{1}, \boldsymbol{\theta}_{2}\right)$, where $\boldsymbol{\theta}_{1}=\left(\boldsymbol{x}_{\mathrm{t}}, \boldsymbol{\phi}_{\mathrm{t}}\right) \in \mathbb{R}^{6}$ and $\boldsymbol{\theta}_{2}=\left(\boldsymbol{\phi}_{\mathrm{a}}, \boldsymbol{\phi}_{\mathrm{wl}}, \boldsymbol{\phi}_{\mathrm{wr}}\right) \in \mathbb{R}^{9}$.

The coordinates used in this study are shown in Fig. 1. The laboratory frame which is fixed to the wind tunnel, is given as $\boldsymbol{e}_{x}, \boldsymbol{e}_{y}$, and $\boldsymbol{e}_{z}$. An inertial coordinate at rest in the laboratory frame is expressed by $\boldsymbol{i}_{\mathrm{I}}, \boldsymbol{j}_{\mathrm{I}}$, and $\boldsymbol{k}_{\mathrm{I}}$, where $\left(\boldsymbol{i}_{\mathrm{I}}, \boldsymbol{j}_{\mathrm{I}}, \boldsymbol{k}_{\mathrm{I}}\right)=\left(-\boldsymbol{e}_{x},-\boldsymbol{e}_{z},-\boldsymbol{e}_{y}\right)$.

The coordinate of the thorax $\left(\boldsymbol{i}_{\mathrm{t}}, \boldsymbol{j}_{\mathrm{t}}, \boldsymbol{k}_{\mathrm{t}}\right)$, which is used to describe the thoracic attitude $\boldsymbol{\phi}_{\mathrm{t}}$, is given by the 1-2-3 Euler angle from the inertial coordinate $\left(\boldsymbol{i}_{\mathrm{I}}, \boldsymbol{j}_{\mathrm{I}}, \boldsymbol{k}_{\mathrm{I}}\right)$. When the flow fields are symmetric with respect to the sagittal plane, the state of the thorax is characterized by the three longitudinal coordinates: $\boldsymbol{x}_{\mathrm{t}}=\left(x_{\mathrm{t}}, y_{\mathrm{t}}, 0\right)$ and $\boldsymbol{\phi}_{\mathrm{t}}=\left(0, \theta_{\mathrm{t}}, 0\right)$. Here, $x_{\mathrm{t}}$ and $y_{\mathrm{t}}$ are, respectively, the streamwise position and the vertical position of the thoracic COM in the laboratory frame $\left(\boldsymbol{e}_{x}, \boldsymbol{e}_{y}, \boldsymbol{e}_{z}\right)$. Note that increase of $x_{\mathrm{t}}$ represents a forward flight slower than $u_{0}$ because $\boldsymbol{x}_{\mathrm{t}}$ is measured in the laboratory frame. The pitching angle about the thoracic $\operatorname{COM} \theta_{\mathrm{t}}$ is the Euler angle $\phi_{\mathrm{t} 2}$ to orient the inertial coordinate $\left(\boldsymbol{i}_{\mathrm{I}}, \boldsymbol{j}_{\mathrm{I}}, \boldsymbol{k}_{\mathrm{I}}\right)$ to the coordinate of the thorax $\left(\boldsymbol{i}_{\mathrm{t}}, \boldsymbol{j}_{\mathrm{t}}, \boldsymbol{k}_{\mathrm{t}}\right)$ by the rotation about the $\boldsymbol{j}_{\mathrm{I}}$ axis. The unit vector $\boldsymbol{i}_{\mathrm{t}}$ is parallel to the line from the thoracic posterior end to the anterior end.

Similarly, the coordinate of each joint $\left(\boldsymbol{i}_{i}, \boldsymbol{j}_{i}, \boldsymbol{k}_{i}\right)$ is given by the 1-3-2 Euler angle from the coordinate of the thorax $\left(\boldsymbol{i}_{\mathrm{t}}, \boldsymbol{j}_{\mathrm{t}}, \boldsymbol{k}_{\mathrm{t}}\right)$. Namely, the coordinate of the thorax coincides with the coordinate of the joint by three composed rotations: the rotations about the $\boldsymbol{i}_{\mathrm{t}}$ axis by $\phi_{i 1}$, about the $\boldsymbol{k}^{\prime}$ axis by $\phi_{i 2}$, and about the $\boldsymbol{j}^{\prime \prime}$ axis by $\phi_{i 3}$. After the composed rotations, the unit vector $\boldsymbol{j}_{\mathrm{wl}}$ is parallel to the line from the left wing joint to the left wing tip, and the unit vector $\boldsymbol{k}_{\mathrm{wl}}$ is normal to the left wing plane (see Ref. 33 for details). Since the flapping motion is assumed to be bilaterally symmetric with respect to the sagittal plane in this study, 4 DOFs out of 9 DOFs in the flapping motion $\boldsymbol{\theta}_{2}$ are independent: $\boldsymbol{\phi}_{\mathrm{a}}=\left(0,0,-\theta_{\mathrm{a}}\right), \boldsymbol{\phi}_{\mathrm{wl}}=(\beta+\pi,-\eta,-\theta)$, and $\boldsymbol{\phi}_{\mathrm{wr}}=(-\beta,-\eta, \theta)$. The flapping angle corresponds approximately to $\beta$, and the flapping angle coincides exactly with $\beta$ when $\eta=\theta=0$. Similarly, the lead-lag angle and the feathering angle correspond approximately to $\eta$ and $\theta$, respectively. Note that the flapping, lead-lag and feathering angles are generally given by combination of $\beta, \eta$ and $\theta{ }^{34}$

Two kinds of experiments with living butterflies were made in a wind tunnel: ${ }^{35}$ a tethered experiment and a free-flight experiment. In the tethered experiment, the inflow velocity was set to be $u_{0}=1.0 \mathrm{~m} / \mathrm{s}$. The thorax of the individual A in Table I was tethered to a tip of a force-torque sensor so that the angle between the long axis of the thorax and the main stream was fixed to $20^{\circ}$ $\left(\theta_{\mathrm{t}}=20^{\circ}\right)$. Because the butterflies do not start to flap if the thoracic angle is too large or too small, the thoracic angle in the tethered experiment is empirically determined so that the butterfly actively flaps. Because of the tethered situation, $\boldsymbol{\theta}_{1}$ is constant in time. The variables needed to construct the joint angles $\boldsymbol{\theta}_{2}$, are expressed by the Fourier series as drawn in Fig. 2. The position and the attitude of the thorax were obtained by optically measuring the positions of two markers made of tiny styrene foams put on the thoracic anterior end and the abdominal joint with three cameras. The joint angles of the abdomen are determined by the positions of the markers put on the thoracic anterior end, the abdominal joint, and the abdominal posterior end. The positions of three markers put on the wing 


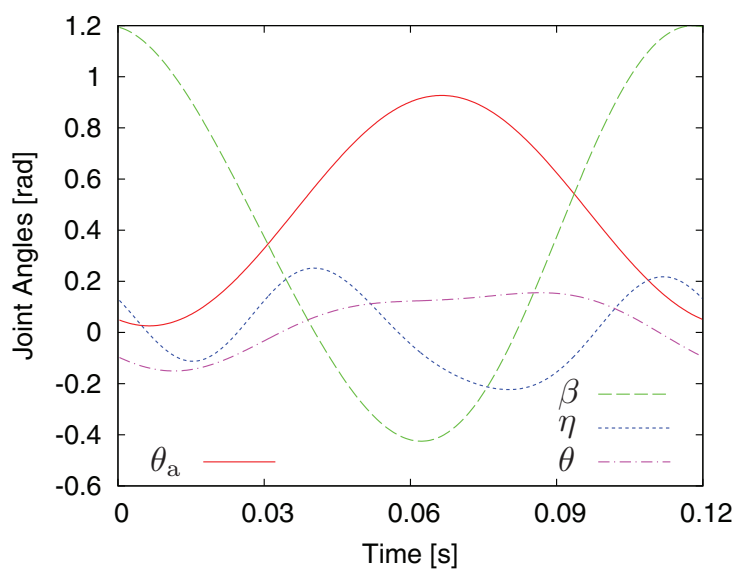

FIG. 2. Observed joint angles of the abdomen and the wings of a tethered butterfly. The abdominal pitching angle, the approximate flapping angle, the approximate lead-lag angle, the approximate feathering angle are denoted by $\theta_{\mathrm{a}}, \beta, \eta$, and $\theta$, respectively.

tip, the trailing edge, and the wing joint were similarly measured. The wing plane is determined by the positions of the three markers. The joint angles of the wing are determined with the wing plane and the thoracic attitude (see Ref. 33 for details). Note that the trajectories were obtained during one specific period without being averaged. For example, the approximate flapping angle $\beta$ is given as $\beta(t)=\sum_{j=0}^{2} C_{\beta j} \cos \left(2 \pi j f t+\varphi_{\beta j}\right)$, where $f=1 / T$ is the fundamental frequency. We define the time origin by the time satisfying $\varphi_{\beta 1}=0$ so that a downstroke is observed during $t \lesssim T / 2$ and an upstroke during $t \gtrsim T / 2$. Similarly, $\theta_{\mathrm{a}}, \eta$, and $\theta$ are expressed by the Fourier series up to the first, the third, and the second orders, respectively. The orders of the truncation are determined so that the Fourier series approximately reproduce the functional forms of the observed joint angles. The abdominal pitching angle $\theta_{\mathrm{a}}$ and the approximate flapping angle $\beta$ are almost in antiphase. Namely, the posterior end of the abdomen goes up during downstrokes. The principal frequency of the approximate lead-lag angles of the wings $\eta$ is twice as large as the fundamental frequency of the approximate flapping angles $\beta$. The feathering motions expressed by $\theta$ are seen to be passively made by the aerodynamic forces. ${ }^{36}$ Note that the flapping motion $\boldsymbol{\theta}_{2}$ varies slightly with both individuals and flaps. In the tethered experiment, the upward and streamwise forces and the longitudinal torque to fix the thorax were measured by the force-torque sensor at the same time as the joint angles $\boldsymbol{\theta}_{2}$ were measured. The measured forces and torque are the sum of the aerodynamic forces, the gravity, and the reaction forces due to the flapping motion.

In the free-flight experiment, the individual B freely flies in the wind tunnel, in which the inflow velocity was set to $u_{0}=1.6 \mathrm{~m} / \mathrm{s}$. In the free-flight experiment, both the thoracic coordinates $\boldsymbol{\theta}_{1}$ and the joint coordinates $\boldsymbol{\theta}_{2}$ were optically measured, although the forces and the torque cannot be measured. The joint angles $\boldsymbol{\theta}_{2}$ are similar to the angles shown in Fig. 2. The thoracic coordinates $\boldsymbol{\theta}_{1}$, which are given by the Fourier series up to the fourth order, are shown in Fig. 3. It is clearly shown that the variation of the vertical coordinate of the thorax $y_{\mathrm{t}}$ is larger than that of the horizontal coordinate $x_{\mathrm{t}}$. Since the free-flight experiment was performed in the wind tunnel where the inflow velocity was $u_{0}$, the difference of the variations can be understood as the trajectory such that butterflies in natural environments fly up and down with almost constant horizontal velocity. The pitching angle of the thorax varies greatly. The variation results mainly from the variation of the aerodynamic torque and that of the reaction torques due to the abdominal flapping motion.

The nondimensional numbers of the flow in the free-flight experiment are listed as follows. The mean wing-tip velocity and the maximal wing-tip velocity are estimated as $4 C_{\beta 1} f y_{\text {tip }}=1.3 \mathrm{~m} / \mathrm{s}$ and $2 \pi C_{\beta 1} f y_{\text {tip }}=2.0 \mathrm{~m} / \mathrm{s}$, respectively. It must be emphasized that the maximal wing-tip velocity is larger than the velocity of the main stream, i.e., the traveling velocity of the butterfly. The Reynolds number evaluated by the mean wing-tip velocity and the wing-tip length is $R e=4 C_{\beta 1} f y_{\text {tip }}^{2} / v_{\text {air }}=4300$. 


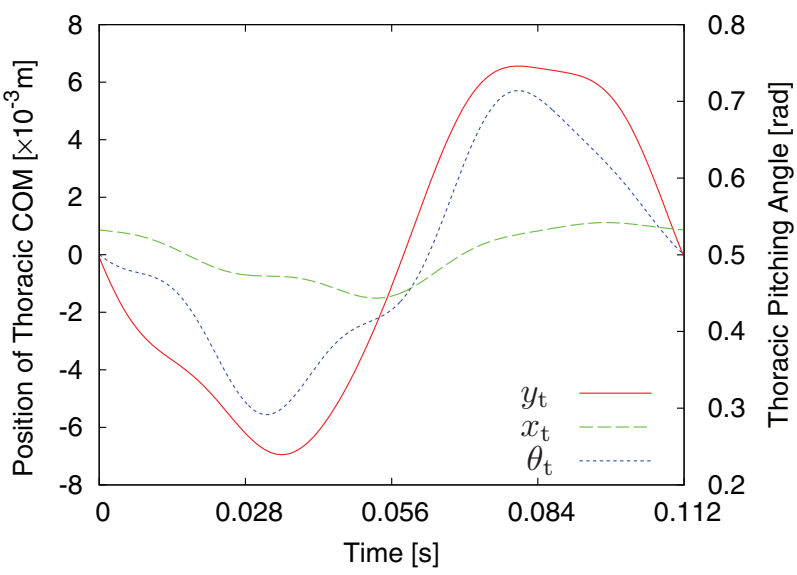

FIG. 3. Observed thoracic coordinates during a free flight. The means of the vertical and horizontal coordinates during the flapping period are set to 0 . The pitching angle is scaled by the right-hand axis.

The Strouhal number in the experiment is $S t=4 C_{\beta 1} f y_{\text {tip }} / u_{0}=0.81$, and the reduced frequency is $k=2 C_{\beta 1} f S_{\mathrm{w}} /\left(u_{0} y_{\text {tip }}\right)=0.20$. The Froude number is $F r=4 C_{\beta 1} f\left(y_{\text {tip }} / g\right)^{1 / 2}=1.9$.

Three kinds of numerical simulations, whose conditions are summarized in Table II, are carried out: a tethered simulation, a prescribed simulation, and a free-flight simulation. The tethered simulation mimics the tethered experiment. In this simulation, the thoracic coordinate $\boldsymbol{\theta}_{1}$ is constant. The COM of the thorax is fixed at $L_{x} / 4$ from the inflow boundary. The thoracic pitching angle is also fixed as $\theta_{\mathrm{t}}=20^{\circ}$. The joint angles of the flapping motion $\boldsymbol{\theta}_{2}$ were obtained by the experimental observation shown in Fig. 2. All the parameters in the simulations are adopted from the experiment except the kinematic viscosity. In the prescribed simulation, the thoracic trajectories in the free-flight experiment shown in Fig. 3 are adopted as the thoracic coordinate $\boldsymbol{\theta}_{1}$. The joint angles are also adopted from the free-flight experiment. In the free-flight simulation, the thoracic motion is numerically solved according to the inverse kinematics in robotics as shown below. The joint angles in this simulation are also adopted from the free-flight experiment. The free-flight simulation is started from the steady periodic state of the prescribed simulation.

The dynamics of the butterfly is expressed by the Euler-Lagrangian equation of motion,

$$
\frac{d}{d t}\left(\frac{\partial L}{\partial \dot{\boldsymbol{\theta}}}\right)-\frac{\partial L}{\partial \boldsymbol{\theta}}=\boldsymbol{\tau},
$$

where $L=K-V$ is the Lagrangian, $K=\dot{\boldsymbol{\theta}} \boldsymbol{M} \dot{\boldsymbol{\theta}} / 2$ is the kinetic energy, and $V$ is the potential energy due to the gravity. Note that the inertia matrix $\boldsymbol{M}(\boldsymbol{\theta}) \in \mathbb{R}^{15 \times 15}$ depends on time through $\boldsymbol{\theta}$. The generalized force $\boldsymbol{\tau} \in \mathbb{R}^{15}$ consists of the aerodynamic force $\boldsymbol{\tau}_{\text {air }}$ obtained from the force $\boldsymbol{F}$ according to the immersed boundary method and the control force $\boldsymbol{\tau}_{\text {cont }}$ applied to the thorax and the joints. For example, the lift, drag, and the pitching moment about the thoracic COM are given

TABLE II. Numerical conditions of three simulations.

\begin{tabular}{lccc}
\hline \hline & Tethered simulation & Prescribed simulation & Free-flight simulation \\
\hline Individual & $\mathrm{A}$ & $\mathrm{B}$ & $\mathrm{B}$ \\
Inflow velocity $u_{0}(\mathrm{~m} / \mathrm{s})$ & 1.0 & 1.6 & 1.6 \\
Kinematic viscosity $v$ & $3 v_{\text {air }}$ & $v_{\text {air }}$ & $2 v_{\text {air }}$ \\
Thoracic coordinates $\boldsymbol{\theta}_{1}$ & Constant & Given by free-flight & Calculated according to \\
& & experiment & Eq. (5) \\
Flapping motion $\boldsymbol{\theta}_{2}$ & Given by tethered & Given by free-flight & Given by free-flight \\
& experiment & experiment & experiment \\
Flapping period $T(\mathrm{~s})$ & 0.12 & 0.112 & 0.112 \\
Time increment $\Delta T$ & $T / 4000$ & $T / 8000$ & $T / 4000$ \\
\hline \hline
\end{tabular}


by the thoracic components of $\boldsymbol{\tau}_{\text {air. }}$. Then, Eq. (2) is rewritten as

$$
\boldsymbol{M} \ddot{\boldsymbol{\theta}}+\dot{\boldsymbol{M}} \dot{\boldsymbol{\theta}}-\frac{1}{2} \frac{\partial}{\partial \boldsymbol{\theta}}(\dot{\boldsymbol{\theta}} \boldsymbol{M} \dot{\boldsymbol{\theta}})+\frac{\partial V}{\partial \boldsymbol{\theta}}=\boldsymbol{\tau}_{\mathrm{air}}+\boldsymbol{\tau}_{\mathrm{cont}}
$$

In all the simulations, the joint angles $\boldsymbol{\theta}_{2}$ are given as functions of time based on the experimental measurements. In the tethered simulation, $\boldsymbol{\theta}_{1}$ is constant in time since the thorax is fixed. In the prescribed simulation, the thoracic trajectory $\boldsymbol{\theta}_{1}$ is also given by the observation in the free-flight experiment. Then, the control forces $\boldsymbol{\tau}_{\text {cont }}$ are obtained according to Eq. (3) in the tethered simulation and the prescribed simulation since all the elements of $\boldsymbol{\theta}=\left(\boldsymbol{\theta}_{1}, \boldsymbol{\theta}_{2}\right)$ are given. On the contrary, in the free-flight simulation, Eq. (3) is solved to obtain the acceleration of the thoracic coordinate $\ddot{\boldsymbol{\theta}}_{1}$. By dividing the variables into the thoracic part and the joint part, Eq. (3) can symbolically be rewritten as

$$
\left(\begin{array}{ll}
\boldsymbol{M}_{11} & \boldsymbol{M}_{12} \\
\boldsymbol{M}_{21} & \boldsymbol{M}_{22}
\end{array}\right)\left(\begin{array}{l}
\ddot{\boldsymbol{\theta}}_{1} \\
\ddot{\boldsymbol{\theta}}_{2}
\end{array}\right)+\left(\begin{array}{l}
\boldsymbol{h}_{1} \\
\boldsymbol{h}_{2}
\end{array}\right)=\left(\begin{array}{l}
\boldsymbol{\tau}_{\text {cont } 1} \\
\boldsymbol{\tau}_{\text {cont } 2}
\end{array}\right),
$$

where

$$
\boldsymbol{h}=\left(\boldsymbol{h}_{1}, \boldsymbol{h}_{2}\right)=\dot{\boldsymbol{M}} \dot{\boldsymbol{\theta}}-\frac{1}{2} \frac{\partial}{\partial \boldsymbol{\theta}}(\dot{\boldsymbol{\theta}} \boldsymbol{M} \dot{\boldsymbol{\theta}})+\frac{\partial V}{\partial \boldsymbol{\theta}}-\boldsymbol{\tau}_{\text {air }}
$$

Because the butterfly cannot control the thorax during free flights directly, $\boldsymbol{\tau}_{\text {cont1 }}=\mathbf{0}$. On the other hand, it is assumed that the butterfly can move the joint angles as they like. Then, $\boldsymbol{\theta}_{2}$ observed in the experiment are assumed to be realized without error by the control of the joints. Therefore, the controlled joint forces can be obtained as $\boldsymbol{\tau}_{\text {cont2 }}=\boldsymbol{h}_{2}-\boldsymbol{M}_{21} \boldsymbol{M}_{11}^{-1}\left(\boldsymbol{M}_{12} \ddot{\boldsymbol{\theta}}_{2}+\boldsymbol{h}_{1}\right)$. Finally, Eq. (4) is solved as

$$
\ddot{\boldsymbol{\theta}}_{1}=-\boldsymbol{M}_{11}^{-1}\left(\boldsymbol{M}_{12} \ddot{\boldsymbol{\theta}}_{2}+\boldsymbol{h}_{1}\right) \text {. }
$$

The time integration of $\boldsymbol{\theta}_{1}$ during the free-flight simulation is made by the second-order AdamsBashforth method according to Eq. (5).

The grid points on the immersed boundary, which are the surface of the thorax and the abdomen and the thin wings, are required to compute the interactive forces according the immersed boundary method. The wing span and the wing chord of each wing are discretized by uniform grids with 128 and 512 points, respectively. The surface of the thorax is discretized by 128 grid points in the long axis direction and 16 in the circumferential direction. The surface of the abdomen is also discretized by 256 grid points in the long axis direction and 16 in the circumferential direction. The effect of the internal mass of the thorax and the abdomen on the forces and torques ${ }^{37}$ is neglected because the spurious forces and torques are much smaller than the real forces and torques acting on the thorax, abdomen and wings.

\section{NUMERICAL RESULTS}

\section{A. Tethered simulation}

In the tethered simulation, the thoracic coordinates $\boldsymbol{\theta}_{1}$ are set to be constant and the thoracic pitching angle is $\theta_{\mathrm{t}}=20^{\circ}$ The data in the tethered simulation and in the prescribed simulation in this section are recorded during one flapping period after the lapse of sufficient time from the initial flap. Note that the forces and torque even during the first flapping period is almost the same as those during other flapping periods except the earliest stage.

The upward and streamwise forces and the longitudinal torque in the simulation and in the experiment are compared in Figs. 4(a)-4(c). The forces and the torque about the COM of the thorax 

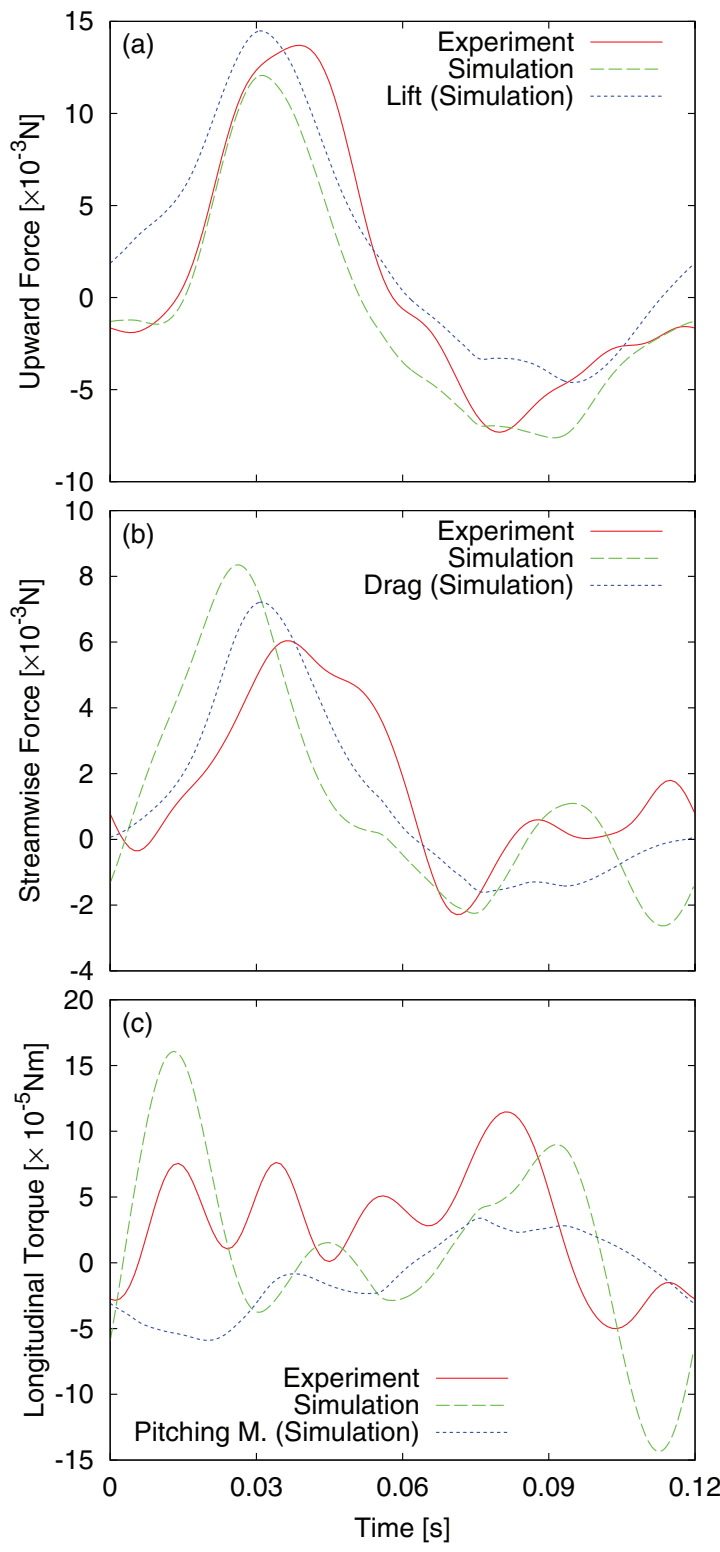

FIG. 4. Upward and streamwise forces and longitudinal torque acting on the thorax of the tethered butterflies in the numerical simulation and the experiment. (a) Upward force, (b) streamwise force, and (c) longitudinal torque. The aerodynamic forces and pitching moment in the simulation are also drawn as the lift, drag, and pitching moment, respectively.

were measured with the force-torque sensor in the tethered experiment. The maximal uncertainty of the static forces in the experimental system is approximately $1 \times 10^{-3} \mathrm{~N}$ for the forces smaller than $2 \times 10^{-2} \mathrm{~N}$. The maximal uncertainty of the static torque is similarly $4 \times 10^{-5} \mathrm{Nm}$ for the torque smaller than $6 \times 10^{-4} \mathrm{Nm}$. The forces and the torque in the tethered experiment correspond to the reaction of the control forces applied to the thorax to fix the thorax $-\boldsymbol{\tau}_{\text {contl }}$ in the tethered simulation. The forces and the torque are composed of the aerodynamic forces, the reaction forces due to the motions of the abdomen and the wings, and the gravity. The lift, the drag and the pitching moment due to the aerodynamic forces, which are the thoracic components of the aerodynamic force $\boldsymbol{\tau}_{\text {airl }}$, are also drawn in Figs. 4(a)-4(c). Note that the forces and the torque both in the simulation and in the experiment are recorded during one period when the joints behave according to the trajectories drawn in Fig. 2 without being averaged. 
The aerodynamic lift accounts for a large fraction of the upward force as shown in Fig. 4(a). The difference between the upward force and the aerodynamic lift results mostly from the gravity $m g$ $=3.3 \times 10^{-3} \mathrm{~N}$. The upward force and the aerodynamic lift reach their maxima around $T / 4$ when the downstroke velocity is maximal. The negative upward force around 3T/4 is also accountable for the negative aerodynamic lift during the downstroke. The mean lift is positive because the angle of attack is positive and the flapping motions are asymmetric, where the time spent on the downstroke is longer than that on the upstroke. The upward force obtained in the numerical simulation agrees with that measured in the experiment. In particular, the agreement is remarkable in the early phase of the downstroke $t<T / 4$.

Similarly to the upward forces, the aerodynamic drag accounts for a large fraction of the streamwise force as shown in Fig. 4(b). The aerodynamic drag is positive during the downstroke, i.e., $t \lesssim T / 2$, and is negative during the upstroke, i.e., $t \gtrsim T / 2$. Since the butterfly flaps the wings with the leading edge up during the upstroke, i.e., $\theta>0$, the aerodynamic thrust, which is caused by the pressure difference between the dorsal and ventral sides of the wings, is obtained during the upstroke. The streamwise force in the numerical simulation qualitatively agrees with that in the experiment.

The aerodynamic pitching moment is positive during the downstroke and negative during the upstroke as shown in Fig. 4(c). The effective points of the application of the aerodynamic forces on the wings lie downstream of the thoracic COM because of the shape of the wings. Therefore, the aerodynamic forces make negative aerodynamic pitching moments about the thoracic COM, i.e., head-down pitching moments during the downstroke. In an opposite manner, the pitching moment due to the aerodynamic forces is positive during the upstroke. Namely, the aerodynamic pitching moment during the upstrokes is a head-up pitching moment.

The gravity acting on the abdomen makes positive longitudinal torque about the thoracic COM. The longitudinal torque due to the gravity acting on the abdomen is roughly $2.5 \times 10^{-5} \mathrm{Nm}$. Note that time variation of the gravitational longitudinal torque is large because of the abdominal motion. Unlike the upward force and the streamwise force, the reaction torques due to the flapping motions rather than the aerodynamic pitching moment consist mostly of the longitudinal torque as shown in Fig. 4(c). In particular, the abdominal motion is responsible for the large difference between the longitudinal torque and the aerodynamic pitching moment around $T / 10$ and the approximate lead-lag motion is for that around 9T/10, when the variation of the approximate lead-lag angle acts like the flapping motion rather than the lead-lag motion.

As shown in Fig. 4(c), the longitudinal torque obtained in the numerical simulation deviates from that measured in the experiment because of the accuracy of the measurements. The discrepancy of the longitudinal torques mostly results from the reaction torques of the flapping motions of the abdomen and the wings. Since the reaction torque strongly depends on $\ddot{\boldsymbol{\theta}}_{2}$, the flapping motions in the experiment must be measured with high resolutions in space and time so that the longitudinal torques in the numerical simulation agree with those in the experiment. Moreover, the longitudinal torque in the experiment requires high accuracy since the value of the longitudinal torque is small. Although the functions in $\boldsymbol{\theta}_{2}$ based on the experimental measurements are not accurate enough, the upward force and the streamwise force reasonably agree with the experimental results. A detailed tuning of the functions might be needed to improve the calculation results.

The flow in the tethered experiment was visualized by smoke released in front of the butterfly so that the smoke intersects with the leading edge of the left wing at the $4 / 5$ length from the wing root to the wing tip in the middle of downstrokes. The smoke during a downstroke is shown in Fig. 5. In the tethered simulation, passive tracers released on the vertical segment of $z=4 y_{\text {tip }} / 5$ on the inflow boundary are used to mimic the smoke in the experiment. The tracers at the middle of the downstroke $t=T / 4$ are shown in Fig. 6. A two-dimensional sheet of the smoke and the tracers are initially given, but they make three-dimensional characteristic patterns in the wakes. Large-scale zig-zag structures of the tracer density caused by the upstrokes and the downstrokes are observed both in the numerical simulation and in the experiment: Figs. 5(b) and 6(b). Similar zig-zag structures were also observed in the wake of a tethered flight of a peacock butterfly. ${ }^{11}$ The sparse regions of the smoke and the tracers indicate stretching due to the flow structures. The dense regions suggest existence of low-pressure vortical structures. Small-scale complex structures of the tracers are also 


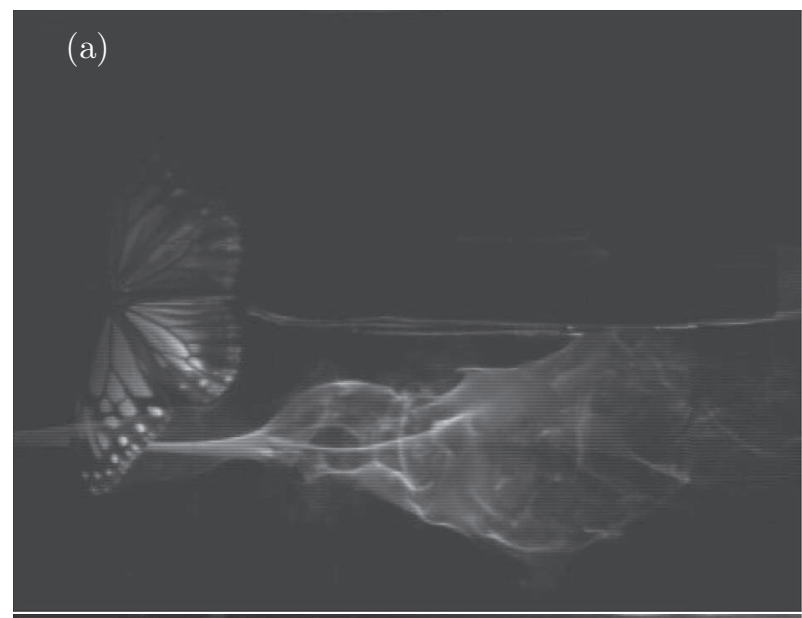

(b)

FIG. 5. Smoke structures in the tethered experiment. The smoke is released on the vertical segment of $z=4 y_{\text {tip }} / 5$ on the inflow boundary. (a) Topview and (b) sideview.

seen. In fact, the tracers follow small- and large-scale vortical structures around, which are identified below.

\section{B. Prescribed simulation}

\section{Forces and torques during flap}

In the prescribed simulation, the thoracic trajectories in the free-flight experiment shown in Fig. 3 are adopted as the thoracic coordinate $\boldsymbol{\theta}_{1}$. Thus, $\boldsymbol{\theta}_{1}$ is not constant in time. The joint angles $\boldsymbol{\theta}_{2}$ are also adopted from the free-flight experiment.

The lift, the drag, and the pitching moment about the COM of the thorax due to the aerodynamic forces are shown in Fig. 7. Similarly to the tethered simulation, the lift is positive during the downstroke and negative during the upstroke. The flapping motions generates lift and the mean lift during one flapping period is close to the gravity $m g=3.3 \times 10^{-3} \mathrm{~N}$. The flapping motion generates the drag during the downstroke and the thrust during the upstroke. The functional form of the feathering angles causes this asymmetry. Because the mean drag is positive, the additional thrust is required for the butterfly to stay against the main stream. The mean pitching moment about the thoracic COM due to the aerodynamic forces is negative, i.e., a head-down moment. The gravity acting on the abdomen produces a positive longitudinal torque about the thoracic COM, i.e., a head-up moment. If the mean pitching moment due to the aerodynamic forces balanced with the mean longitudinal torque due to the gravity, the periodic flight should be realized. Note that the signs of the mean reaction torques due to the flapping motion are not directly related to the periodicity 

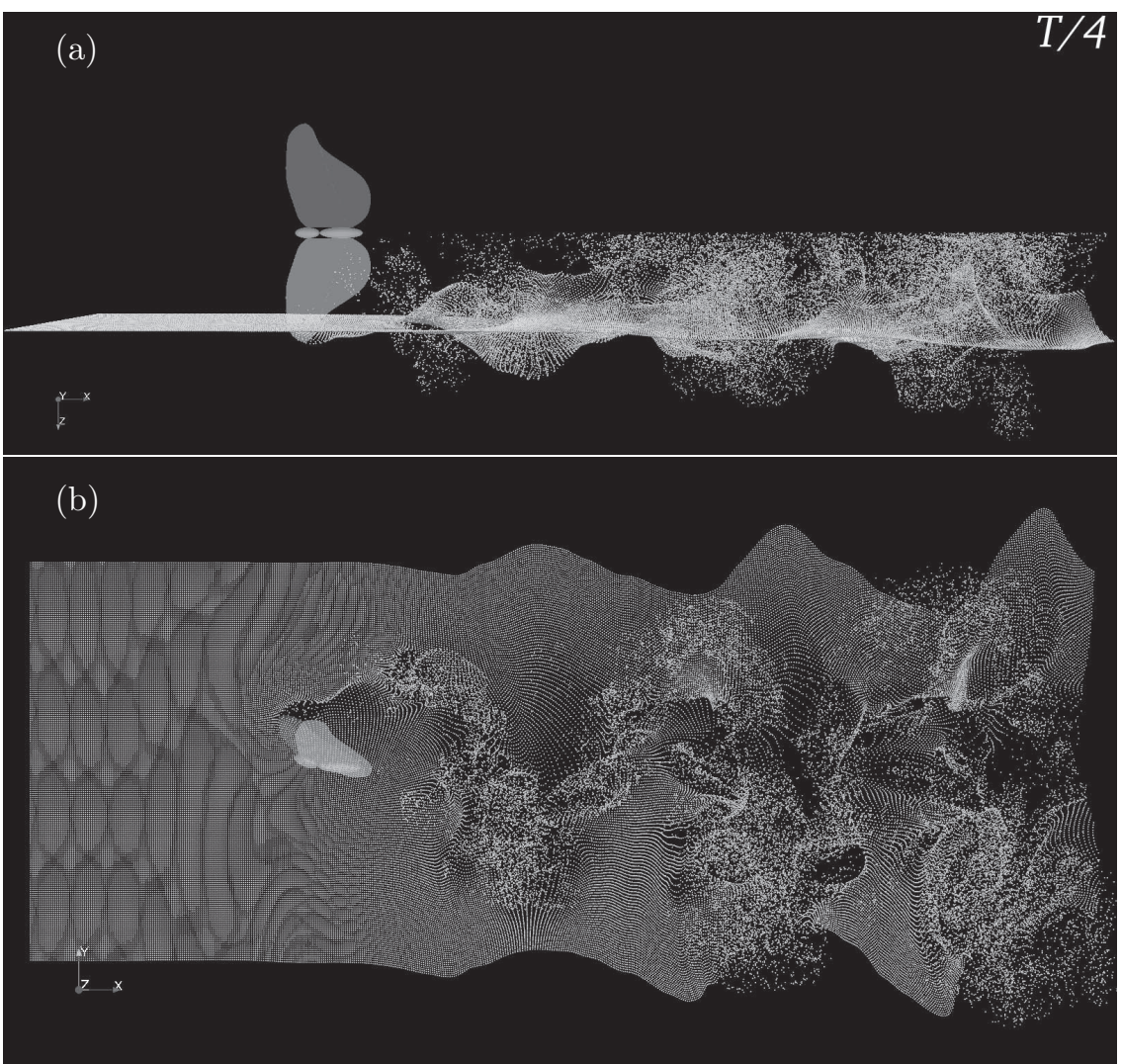

FIG. 6. Passive tracers in the tethered simulation. (a) Topview and (b) sideview.

of the flights since the torques applied to the joints are internal torques for the butterfly motion. It should also be noted that the forces in the prescribed simulation are larger than those in the tethered simulation mainly because the individual B used in the prescribed simulation has the wings larger than the individual A used in the tethered simulation has.

The forces and the longitudinal moment due to the extra control $\boldsymbol{\tau}_{\text {cont1 }}$, which are required so that the thoracic trajectory $\boldsymbol{\theta}_{1}$ in the simulation traces that observed in the free-flight experiment,

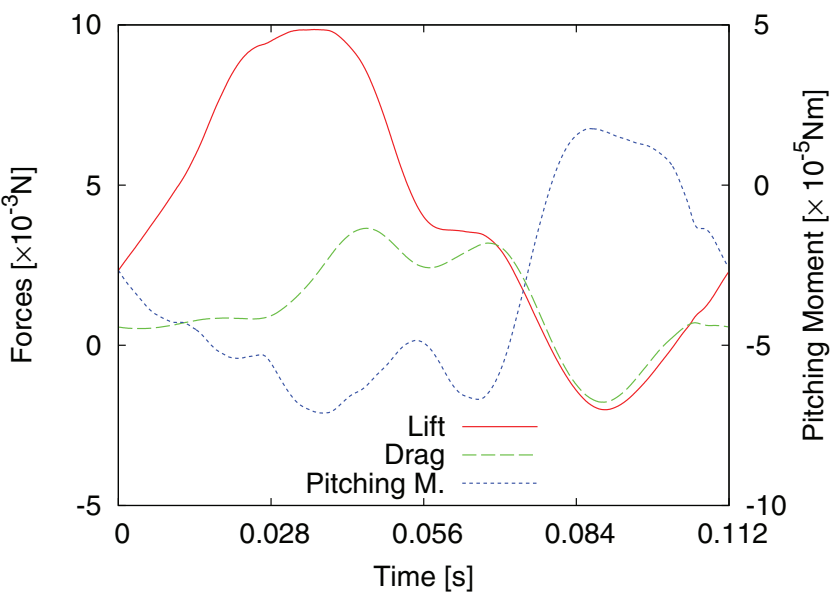

FIG. 7. Lift, drag, and pitching moment due to aerodynamic force in the prescribed simulation. The pitching moment is scaled by the right-hand axis. 


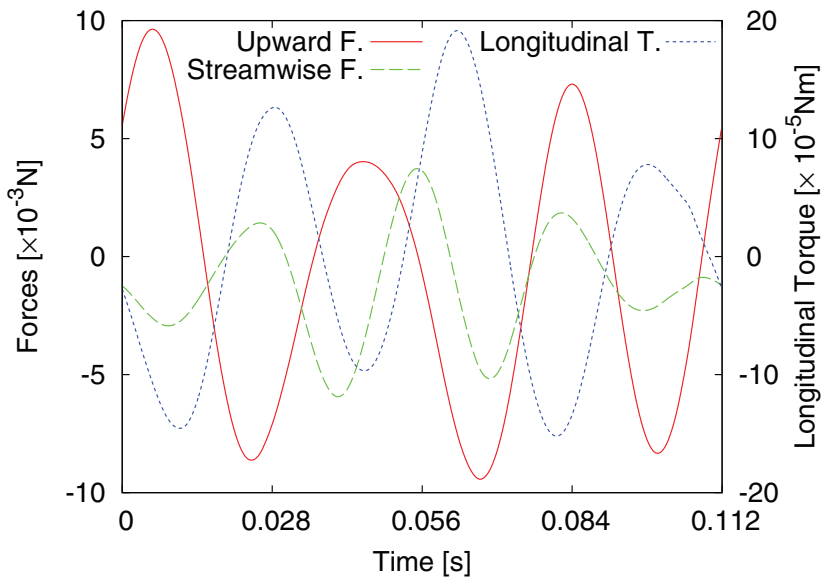

FIG. 8. Upward and streamwise forces and longitudinal torque due to extra control applied to the thorax in the prescribed simulation. The longitudinal torque are scaled by the right-hand axis.

are shown in Fig. 8. The upward and streamwise forces given by the extra control term are as large as the lift and the drag, respectively. On the other hand, the longitudinal torque due to the extra control is thrice as large as the aerodynamic pitching moment. In this simulation, the external control forces and torque comparable to the calculated aerodynamic forces and torque are still required. It suggests that the flight of the butterfly is realized upon delicate control mechanisms. Because third or higher harmonics account for the extra control forces in Fig. 8, more accurate measurement of the trajectories $\boldsymbol{\theta}_{1}$ and $\boldsymbol{\theta}_{2}$ in the experiment would make $\boldsymbol{\tau}_{\text {cont1 }}$ smaller.

The torques applied to the joints between the thorax and the abdomen and between the thorax and the left wing are shown in Fig. 9. The pitching angle of the abdomen $\theta_{\mathrm{a}}$, the approximate flapping angle $\beta$, the approximate lead-lag angle $\eta$, and the approximate feathering angle $\theta$ of the left wing correspond to the components of the Euler angles of the joints as $\theta_{\mathrm{a}}=-\phi_{\mathrm{a} 3}, \beta=\phi_{\mathrm{wl}}$ $-\pi, \eta=-\phi_{\mathrm{wl} 2}$, and $\theta=-\phi_{\mathrm{wl} 3}$. Then, the torques corresponding to $\theta_{\mathrm{a}}, \beta, \eta$, and $\theta$ are defined by each components of the generalized torque as $\tau_{\theta_{\mathrm{a}}}=-\tau_{\mathrm{a} 3}, \tau_{\mathrm{wl} \beta}=\tau_{\mathrm{wl} l}, \tau_{\mathrm{wl} \eta}=-\tau_{\mathrm{wl} 2}$, and $\tau_{\mathrm{wl} \theta}$ $=-\tau_{\mathrm{wl} 3}$. Note that these torques applied to the joints are internal torques while the extra control forces are external forces. The control torques of the abdomen $\tau_{\theta_{\mathrm{a}}}$ and the approximate flapping angle $\tau_{\mathrm{wl} \beta}$ are larger than those of the approximate lead-lag angle $\tau_{\mathrm{wl} \eta}$ and the feathering angle $\tau_{\mathrm{wl} \theta}$. These results fit with our observations that the butterflies actively control the abdominal motion and the flapping angles. On the other hand, the variation of the feathering angle is passively made by

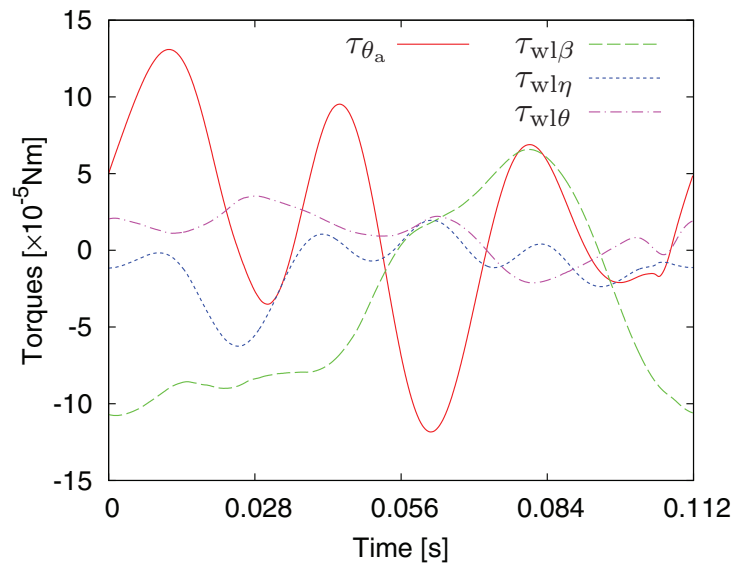

FIG. 9. Torques applied to the joints of the abdomen $\tau_{\theta_{\mathrm{a}}}$ and of the left wing $\tau_{\mathrm{wl} \beta}, \tau_{\mathrm{wl} \eta}$ and $\tau_{\mathrm{wl} \theta}$ in the prescribed simulation. 


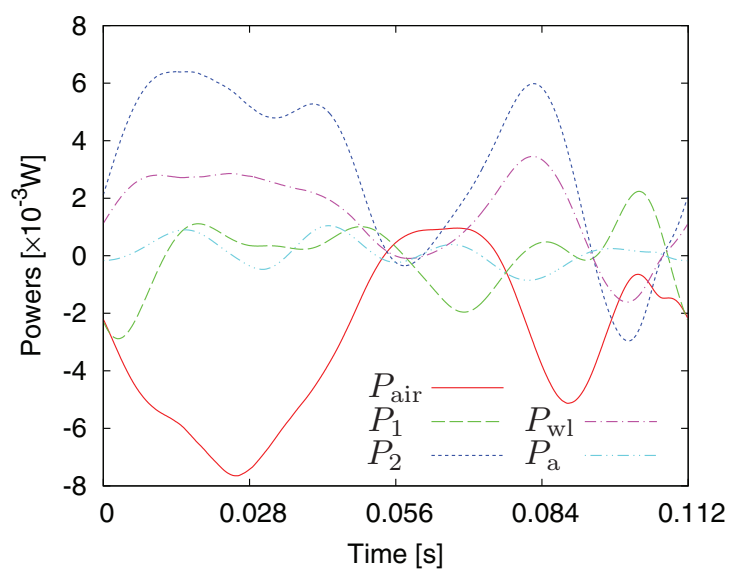

FIG. 10. Power due to aerodynamic force $P_{\text {air }}$, extra control $P_{1}$, and joint control forces $P_{2}$ in the prescribed simulation. The power due to the joint control is the sum of those due to the abdominal and wing joints, i.e., $P_{2}=P_{\mathrm{a}}+P_{\mathrm{wl}}+P_{\mathrm{wr}}$.

the aerodynamic forces. ${ }^{36}$ It is expected that the butterflies can control the lead-lag angle little since monarch butterflies related to the chestnut tiger butterflies have only small muscles to control the lead-lag angle. ${ }^{38}$ These biological facts are consistent to the torques drawn in Fig. 9.

Power done by air, by the torques of the joints, and by the extra control forces are shown in Fig. 10. Power done by air $P_{\text {air }}=\boldsymbol{\tau}_{\text {air }} \cdot \dot{\boldsymbol{\theta}}$ is almost always negative, showing that the butterfly moves the joints against the air. The power done by control of the joints, $P_{2}=\boldsymbol{\tau}_{\text {cont2 }} \cdot \dot{\boldsymbol{\theta}}_{2}$, is the sum of the power done by all the three joints, i.e., $P_{2}=\sum_{i \in\{\mathrm{a}, \mathrm{wl}, \mathrm{wr}\}} P_{i}$, where $P_{i}=\boldsymbol{\tau}_{i} \cdot \boldsymbol{\phi}_{i}$. The power done by the left wing $P_{\mathrm{wl}}$, which is almost identical to the power done by the right wing $P_{\mathrm{wr}}$ owing to the bilaterally symmetrically flapping motions, is positive in the middle of the downstroke and the upstroke. Then, the power done by the control of the joints $P_{2}$ is positive in the middle of the downstroke and the upstroke. Because $P_{2} \approx 2 P_{\text {wl }}$, the power done by the control of the joints comes mainly from that done by the joints of the wings. It is of interest that the power done by the abdominal joint is much smaller than that by the joints of the wings although the torques applied to the joints are comparable. Thus, the control with the abdominal motion is energy-efficient. The power done by the extra control forces, $P_{1}=\boldsymbol{\tau}_{\text {cont1 }} \cdot \dot{\boldsymbol{\theta}}_{1}$, shown also in Fig. 10 is much smaller than that done by the control of the joints $P_{2}$. It also supports that the control required to steady periodic flights can be done energy-efficiently by the abdominal motions.

The powers of various free-flying insects including tobacco hornworm belonging to Lepidoptera are roughly estimated as $10^{2} \mathrm{~W} / \mathrm{kg}$ muscle, and thoracic muscle mass accounts for one third of body mass of Lepidoptera. ${ }^{27}$ This results in $10^{-2} \mathrm{~W}$ in the butterfly with $1 \times 10^{-4} \mathrm{~kg}$ thoracic muscle mass, which is of the same order as $P_{2}$ in Fig. 10.

\section{Vortical structures}

The vortical structures of the flow field are shown in Fig. 11. The low-pressure vortices are identified by the $Q$-criterion, ${ }^{39}$ i.e., the second invariant of the velocity gradient tensor $Q$ $=\nabla^{2} p /\left(2 \rho_{\text {air }}\right)$. The isosurface of $Q=1 \times 10^{5} \mathrm{~s}^{-2}$ at the middle of the downstroke $t=T / 4$ are drawn in Fig. 11. Note that the slight noise seen around the wings is caused by the visualization technique and it does not affect the forces and the torques acting on the butterfly. The three-dimensional vortical structures and their directions are schematically drawn in Fig. 12. The movie of the vortical structures during one flapping period can be downloaded from Ref. 40. For visibility of the movie, the isosurface of $Q=1.2 \times 10^{5} \mathrm{~s}^{-2}$ is drawn in red over the entire computational domain in the movie while the isosurface of $Q=3 \times 10^{4} \mathrm{~s}^{-2}$ is drawn in blue only for the structures generated in the previous flap.

The large-scale zig-zag structures, similar to those in Figs. 5(b) and 6(b), are recognized also in Fig. 11(b). Since the flow is turbulent, small-scale structures are observed and the bilateral symmetry 
(a)

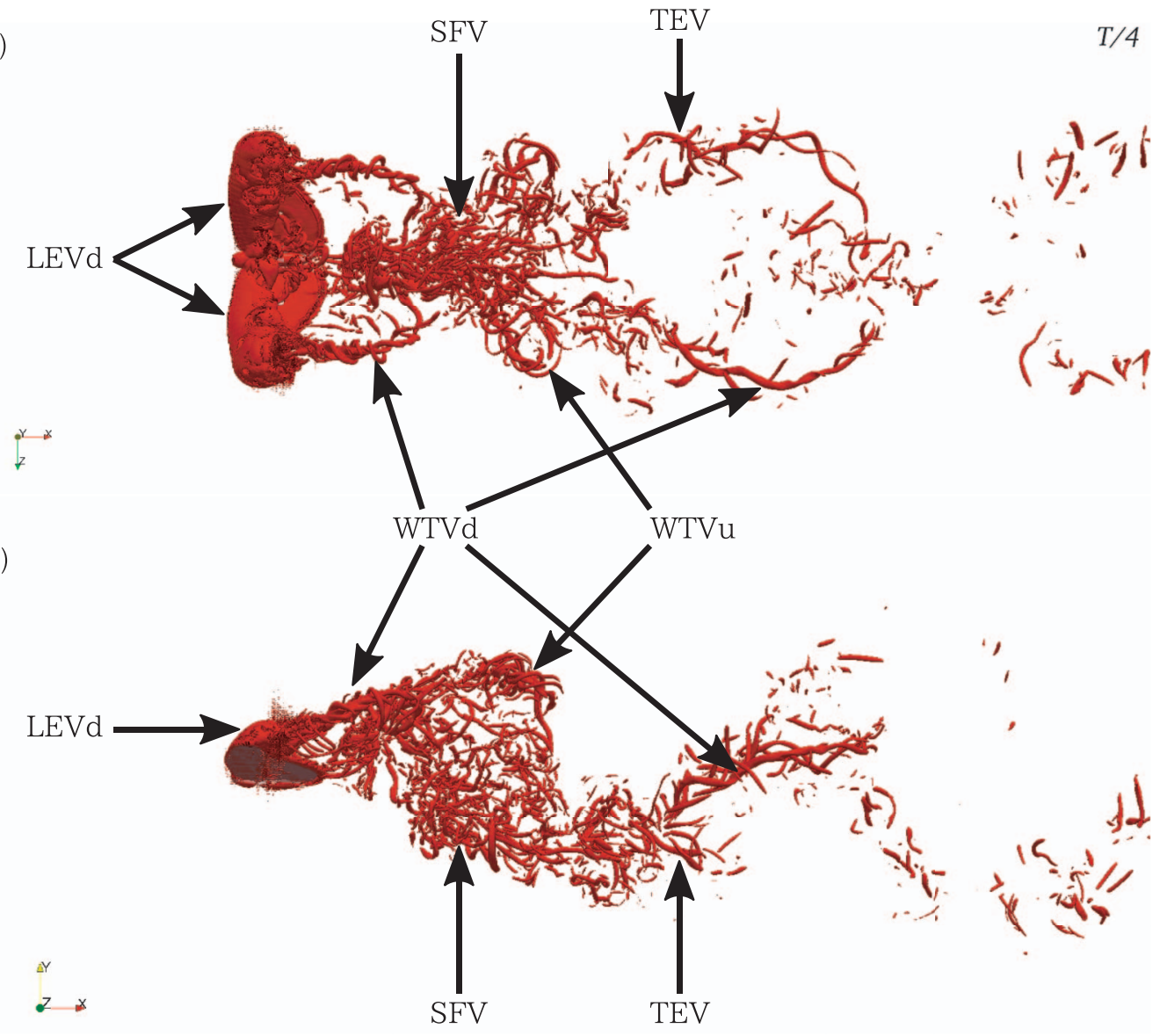

FIG. 11. Vortical structures visualized by the $Q$-criterion, i.e., the second invariant of velocity gradient tensor in the prescribed simulation. The isosurface of $Q=1 \times 10^{5} \mathrm{~s}^{-2}$ is drawn. (a) Topview and (b) sideview.

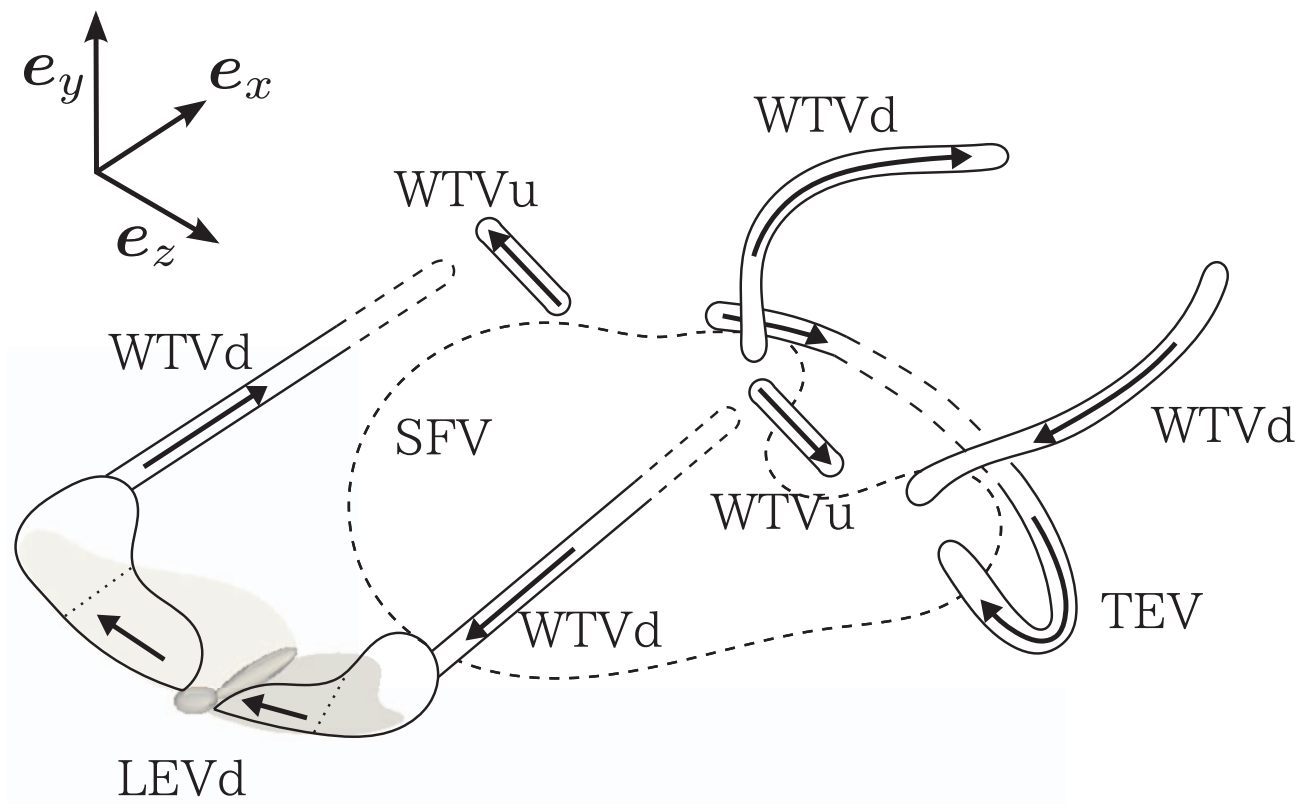

FIG. 12. Schematic illustration of the three-dimensional vortical structures. The directions of the coherent vortices are expressed by the arrows. 
of the flow field is slightly broken. Even large-scale coherent structures are not similar to a chain of coupled vortices proposed by in Ref. 11. In fact, the large-scale coherent structures in the wake are similar to those of a low-aspect-ratio flapping foil ${ }^{10}$ and the flow structures are significantly complex. Small-scale complex vortices are dissipated or merged into large-scale coherent vortices, and then only the large-scale coherent vortices survive in the far field. The tracers in Fig. 6 mainly follow the strong vortices.

The generation process of such zig-zag vortical structures is as follows. Several thin swirling vortices start from each wing tip during a downstroke. The thin swirling vortices twist immediately after the separation from each wing tip and form a wing-tip vortex during the downstroke (WTVd). As swept in the downstream, the thin vortices are merged by the viscosity into a coherent vortex. Because the directions of the vorticities of the WTVds starting from the left and right wing tips are, respectively, $-x$ and $+x$ (see Fig. 12), the fluid particles between the two WTVds are advected to the downward direction. Then, the WTVds generate lift as the reaction of inducing the downward flow. Wing-tip vortices during upstrokes (WTVu) are also made; the vorticities of the left and right WTVus are aligned close to the negative and positive $y$ direction, respectively. Therefore, the flow along the positive $x$ direction is enhanced, which generates the thrust. The thrust due to the WTVus is smaller than the lift due to the WTVds because the WTVus are less coherent than the WTVds and the WTVus induce weaker airflow. Trailing-edge vortices (TEV) released at the transitions from downstrokes to upstrokes are barely visible in the wake. The TEV makes the flow enclosed in the TEV downward and forward. Then, the TEVs generate the lift and the drag on the butterfly, and the generation of the lift and the drag due to the TEV is seen during $T / 2 \lesssim t \lesssim 5 T / 8$ in Fig. 7 .

Among these coherent structures in the wake, leading-edge vortices during downstrokes (LEVd), which are the vortices generated by the separation from the entire leading edges, are formed on the dorsal side of the wings. The separation on the leading edges makes complex secondary flow vortices (SFV) below the LEVds (see Fig. 13(a) below). The SFVs are released around the transitions from downstrokes to upstrokes in the movie (Ref. 40). The vortical directions of the SFVs are not uniformly aligned. These fine complex flows are smoothened by the viscosity, and they disappear as they are advected downstream.

The mechanisms of the formations of the vortical structures are clarified by the visualization of near-field flows. The vortical structures as well as the aerodynamic forces which act on the wings at three distinct times are depicted in Figs. 13(a), 13(c), and 13(d). The aerodynamic forces $\boldsymbol{F}\left(\boldsymbol{X}_{n}, t_{n+1 / 2}\right)$ consisting of the pressure forces and the viscous forces are drawn only on the left wing while the isosurface of $Q=2.5 \times 10^{5} \mathrm{~s}^{-2}$ is drawn only on the right side. Note that the forces along the edges of the wings appear to be large, but the integral of the forces along the edges are smaller than that on the rest of the wing.

To identify structures of strong vortices at the middle of the downstroke, we show in Fig. 13(b) two groups of streamlines: the streamlines in a group start from the points on the line segment in front of the leading edge, while the streamlines in the other group start from the points on the line segment located in the middle of the left wing. Both starting segments are parallel to the wing plane and just above the plane. The spanwise vorticities $\omega_{z}$ and the streamwise vorticities $\omega_{x}$ are, respectively, drawn on a cross-section on the right wing and on another cross-section in the downstream in Fig. 13(b). Both cross-sections are normal to the $x$ direction. A movie of the near-field vortical structures during one flapping period can be downloaded from Ref. 40. The isosurface of $Q=2.5$ $\times 10^{5} \mathrm{~s}^{-2}$ is drawn in the movie.

In the middle of the downstroke, $t \approx T / 4$, the LEVds separate from the leading edges on the dorsal side of the wings as shown in Fig. 13(a). The streamlines starting from the segment in front of the leading edge in Fig. 13(b) fall into two categories based on their patterns. The streamlines starting from near the thorax make a thick tubular structure in the middle of the wing and go through the wing to the ventral side. On the other hand, the streamlines starting from near the wing tip go through the wing to the ventral side near the leading edge. The behaviors of the streamlines clearly indicate two kinds of the LEVds: one is the LEVd near the wing root and the other is the LEVd near the wing tip. The LEVds have the vorticities in the negative $z$ direction. The LEVd near the wing root appears as a sheet-like structure where the negatively large $\omega_{z}$ are localized on the upstream cross-section. The LEVd near the wing tip contains small vortical structures. The 

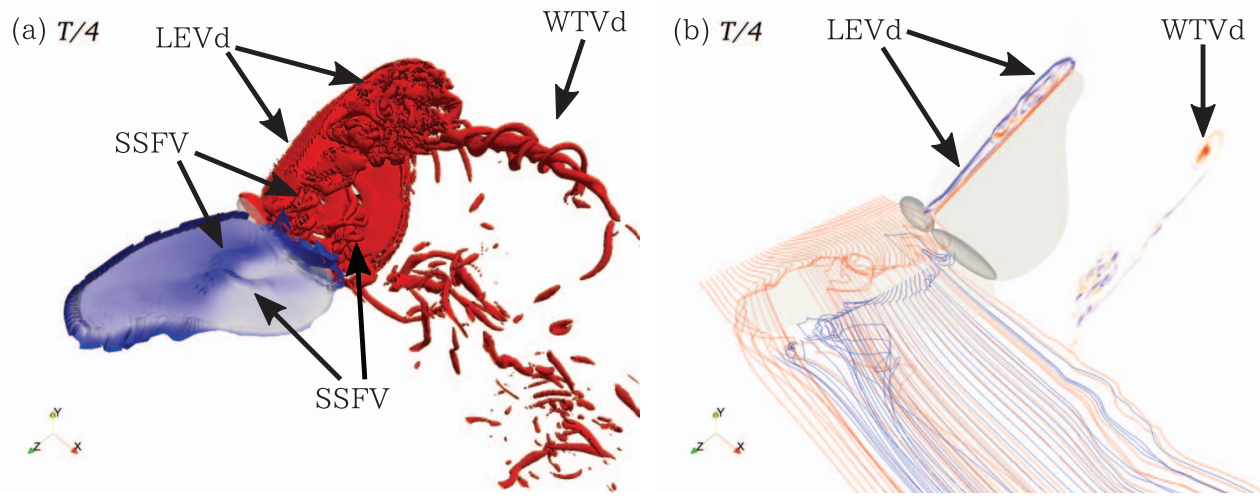

(c) $5 T / 8$

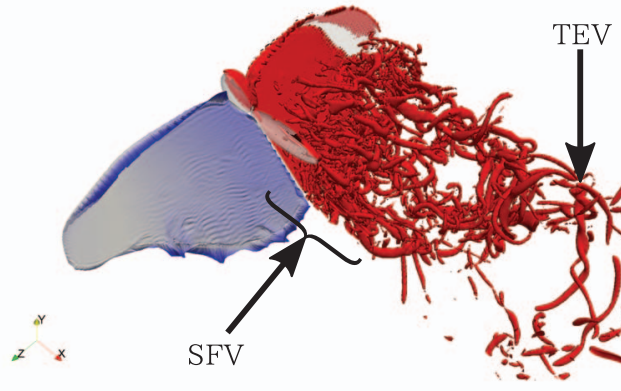

(d) $7 \mathrm{~T} / 8$

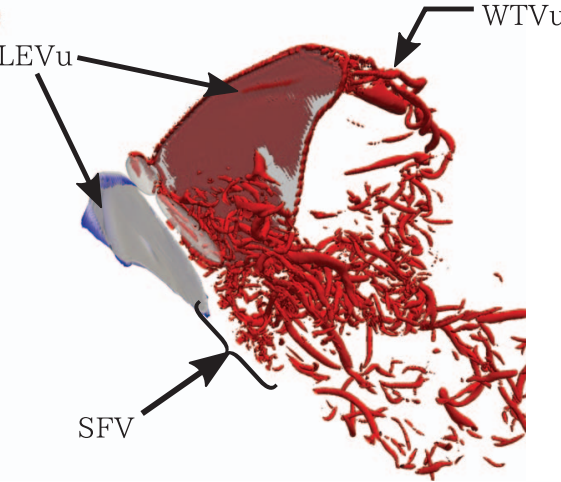

FIG. 13. Near-field structures viewed from above and behind in the prescribed simulation. The forces and the isosurface of $Q=2.5 \times 10^{5} \mathrm{~s}^{-2}$ are drawn. The streamlines starting from two segments are drawn at $t=T / 4$. The spanwise vorticities $\omega_{z}$ on the right wing and the streamwise vorticities $\omega_{x}$ in the downstream are also drawn on the corresponding cross-section at $t=T / 4$. (a) Forces and $Q$-criterion at $t=T / 4$, (b) streamlines and vorticities at $t=T / 4$, (c) forces and $Q$-criterion at $t=5 T / 8$, and (d) forces and $Q$-criterion at $t=7 T / 8$. The magnitudes and the direction of the forces are, respectively, expressed by density (density of blue) and by the displacement from the wing. The forces and the streamlines are shown on the left side while the isosurface of $Q$ and the vorticities are shown on the right side.

emergence of the two kinds of the LEVds are reported in hawkmoth's hovering flight. ${ }^{41}$ The flow structures due to the LEVd near the wing root on the wing in the middle of the downstrokes are much similar to the flow behind the high angle-of-attack inclined plates as shown in Fig. 13(a). Since the larger value of the $Q$-criterion than that in Fig. 11 is adopted to identify the vortical structures, the secondary flow vortices below the LEVd near the wing root, which are seen as the SFVs in the downstream in Fig. 11, can be seen clearly. The vortical directions of the SFVs are not uniformly aligned, but the mean vortical direction of the SFVs on the wings around $t=T / 4$ are found in the near-field movie to be $-z$, the same direction as that of the LEVds. Two relatively strong vortices among other SFVs (SSFV) are found on the wing during the downstroke. The SSFVs have the spanwise vorticities. The spanwise flow of the LEVd near the wing root goes to the wing tip, and the LEVd near the wing tip causes the vortex breakdown. ${ }^{42}$ It is of interest that the boundary of the two kinds of the LEVds corresponds to the widening region of the wings on the trailing edge. The shape of the wings might be advantageous for the WTVds not to interfere with the wings.

The WTVds twist inside the break-down region of the LEVds near the wing tips and the thin swirling vortices make a multiplex helical structure. Each swirling vortex starting from the right wing tip has $\omega_{x}>0$ (see the vorticities on the downstream cross-section in Fig. 13(b)). The direction of the multiplex helical structure starting the right wing tip is left-handed since newly generated swirling flow in the upstream is stronger than that in the downstream. The WTVds are identified also by the outer streamlines starting from the segment in the middle of the wing shown in Fig. 13(b). 
The distribution of the forces is closely correlated with the distribution of $Q$. The pressure inside the vortical structures identified by $Q$ is smaller than that outside the structures. The positive $Q$ appears on the dorsal side because the pressure of the dorsal side of the wings is smaller than that of the ventral side during the downstrokes. By the same token, all the forces except those on the trailing edges point upward, and act as lift on the wings. The large local lifts are found below the separated LEVd on the anterior half corresponding to a forewing, while the local lifts on the posterior half corresponding to a hindwing are small. The enhancement of the lift due to the LEVds were observed similarly in hawkmoth's hovering flight. ${ }^{15}$ In particular, the larger local lifts are found below the SSFVs. Because the pressure inside the tubular SSFVs is smaller, the pressure difference between the dorsal side and the ventral side is larger near the SSFVs than in other regions. The pitching moment strongly depends on the position of the vortical structures on the wings such as the SSFVs, and therefore the vortical structures largely affect the thoracic pitching angle of the butterfly.

At the early phase of the upstroke $t=5 T / 8$ shown in Fig. 13(c), the isosurfaces of positive $Q$ appear near the wing roots on the dorsal side and near the wing tips on the ventral side. Corresponding to the distribution of $Q$ on the wings, the forces on the wings near the wing root point to the dorsal side and those near the wing tips to the ventral side. The leading-edge vortices during upstrokes (LEVu) develop near the wing tips on the ventral side (which is not seen clearly in Fig. 13(c)). The semicircular TEV followed by the SFVs can be recognized in Fig. 13(c). The TEV barely survives in the downstream in Fig. 11, because the direction of the vorticities of the TEV is circumferentially aligned unlike the SFVs although the swirling flow due to the TEV is not so strong as the WTVds. The LEVds on the dorsal side become weak around the transitions from downstrokes to upstrokes. In addition, the thoracic pitching angle and the feathering angle is large in the early phase of upstrokes. Then, the SFVs are released to the downstream. The forces near the trailing edges largely fluctuate because some of the SFVs pass over the trailing edges.

At the late phase of the upstroke $t=7 T / 8$ shown in Fig. 13(d), the isosurfaces of the positive $Q$ appear on the ventral side and most forces on the wings point to the ventral side. Because the thoracic pitching angle is large and the feathering angle is positive, the forces during the upstroke act on the butterfly as the thrust. In particular, large forces are found near the wing tip. The LEVu on the ventral side, which deviates from the leading edge near the wing tip because of the delayed separation, generates the large forces. The WTVu is weakly connected to the LEVu near the wing tip on the ventral side. The direction of the vorticity of the WTVu starting from the right wing tip is close to $+y$ and that starting from the left wing tip is to $-y$. The flow between the WTVus are accelerated in the positive $x$ direction. Then, the WTVus make thrust. It is of interest that the turbulent small structures, the SFVs, are cleared on the wings at $t=7 T / 8$. The mechanism of the clearance of the turbulent structures should be necessary for the steady periodic flapping flights.

The behaviors of the LEVds in this simulation are much similar to those in hawkmoth's hovering flight ${ }^{41}$ in spite of different species and of different flapping patterns. On the other hand, the vortical structures on the wings are much more complex although the Reynolds numbers are in the same range. The elastic deformation of the wings more or less suppresses the turbulent structures, and slightly affects the resulting forces. ${ }^{43,44}$ However, even with the deformable wings, the three-dimensional flapping flights for $R e \approx 4000$ would generate the complex vortical structures. The fine numerical grids are required for the turbulent structures to be resolved. These complex vortical structures on the wings and their clearance are important features of butterflies' forward flights.

To compare the flapping flights with gliding flights, we performed a simulation of gliding flight by fixing the attitude and the joint angles of the butterfly as those at $t=T / 4$ in the prescribed simulation. In the steady state, the lift, the drag and the pitching moment are approximately 1 $\times 10^{-4} \mathrm{~N}, 4 \times 10^{-4} \mathrm{~N}$, and $2 \times 10^{-6} \mathrm{Nm}$, respectively. The mean wing-tip velocity in the flapping flight is approximately $1.3 \mathrm{~m} / \mathrm{s}$, and the velocity of other parts of the wing is smaller than it. Although the relative wing velocity in the flapping flight is smaller than the inflow velocity, the mean lift in the flapping flight $3 \times 10^{-3} \mathrm{~N}$ in Fig. 7 is roughly 30 times larger than that in the gliding flight. Similarly, the flow is laminar, and no coherent vortical structures are found in the gliding simulation unlike the gliding flight of a simplified model in Ref. 45. Therefore, the flapping motions play a crucial role in forward flights of butterflies. 


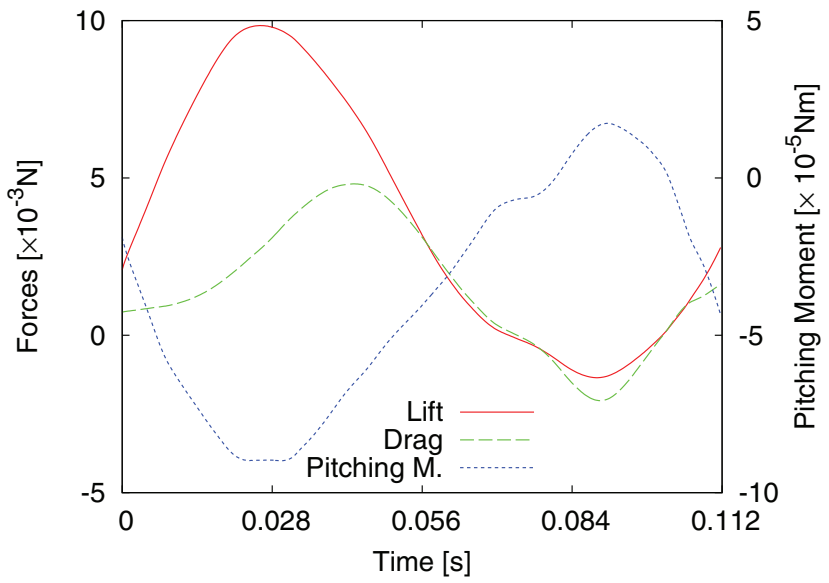

FIG. 14. Lift, drag, and pitching moment due to aerodynamic force during the first free-flight flapping period.

\section{DISCUSSION: FREE-FLIGHT SIMULATION}

After the steady periodic prescribed flight is achieved, the extra control forces applied to the thorax in the prescribed flight are removed at $t=0$, i.e., $\boldsymbol{\tau}_{\text {cont } 1}=\mathbf{0}$ for $t \geq 0$, and then the freeflight simulation is performed. Here, the time when the extra control forces are removed is chosen the time at the transition from an upstroke to a downstroke, although the free-flight trajectories should depend on the phases of the flapping motions when the extra control forces start to be removed.

While the lift, the drag and the pitching moment due to the aerodynamic forces during the first flapping period in the free-flight simulation are shown in Fig. 14, the thoracic coordinates are shown in Fig. 15. The aerodynamic pitching moment during the downstroke is negative while the abdominal motion and the gravity acting on the abdomen make a positive longitudinal torque on the thorax. The longitudinal torque due to the gravity acting on the abdomen is roughly $3 \times 10^{-5} \mathrm{Nm}$. Because the absolute value of the negative pitching moment during the downstroke is smaller on average than the longitudinal torque due to the gravity acting on the abdomen, the thoracic pitching angle during the downstroke in Fig. 15 decreases little, and the minimal thoracic pitching angle is larger than that of the observed trajectory shown in Fig. 3. The thoracic pitching angle at the end of the first flap $t=T$ is much larger than the initial pitching angle. Namely, the butterfly is going to be turned upward and to fall. The lift in the free-flight simulation is larger than that in the prescribed simulation and is large enough to sustain the altitude against the gravity. Then, the butterfly maintains its altitude

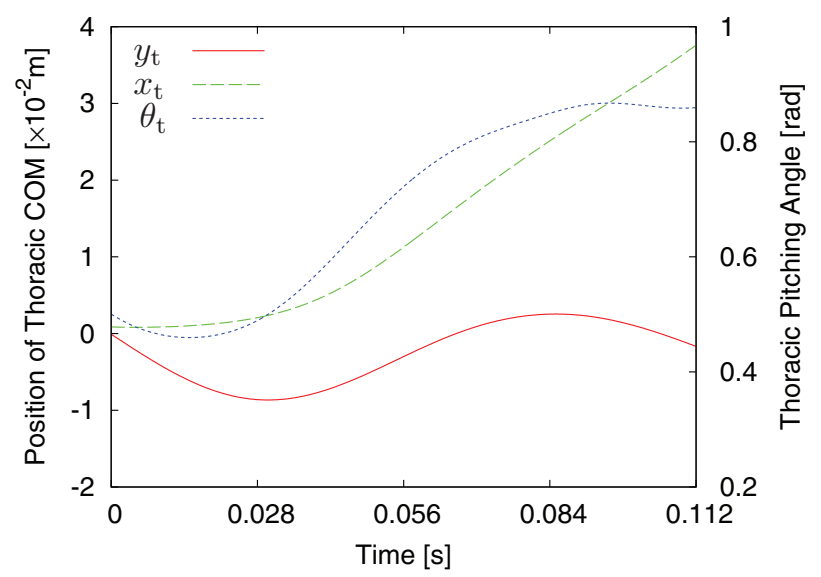

FIG. 15. Thoracic coordinates during the first free-flight flapping period. 


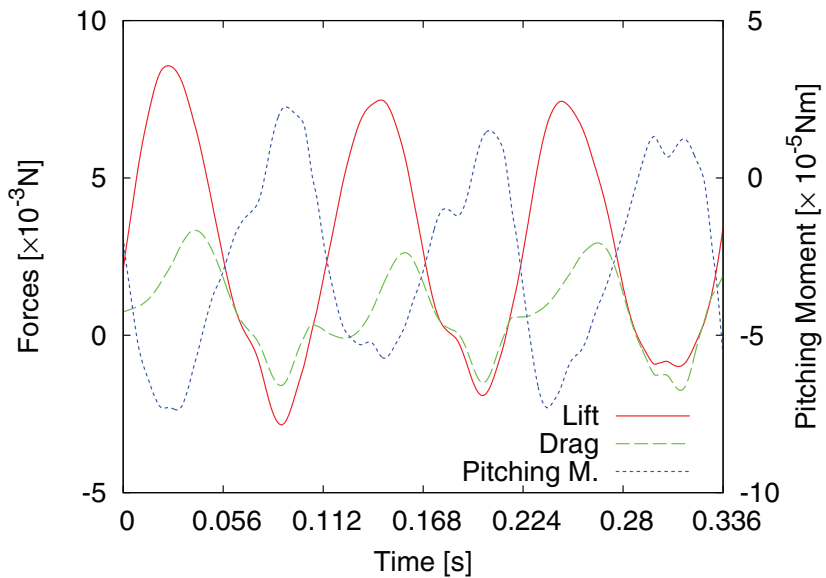

FIG. 16. Lift, drag and pitching moment due to aerodynamic force during the three free-flight flapping periods with the smaller abdominal mass $3 m_{\mathrm{a}} / 4$.

after one flapping period. On the other hand, the drag during the downstroke is large and the thrust during the upstroke is small. Therefore, the butterfly is swept downstream in the frame fixed to the wind tunnel. Note that the butterfly is still going forward at approximately $1.1 \mathrm{~m} / \mathrm{s}$ at $t=T$ in the frame of reference traveling with the main stream because the horizontal velocity in the laboratory frame at $t=T$ is about $0.5 \mathrm{~m} / \mathrm{s}$.

The lengths and the total mass of the butterfly were measured at the end of the free-flight experiment. However, the masses of each parts are statistically estimated. During the experiment, the values of the abdominal mass largely vary half to twice as large as the mean abdominal mass. In consideration of the variation of the abdominal mass, another free-flight simulation is performed, where a reduced abdominal mass $3 m_{\mathrm{a}} / 4$ is adopted. The inertial tensor of the abdomen is also multiplied by $3 / 4$. In the free-flight simulation with the smaller abdominal mass, the total mass is also reduced to $2.83 \times 10^{-4} \mathrm{~kg}$ because the masses of the thorax and the wings the same as those in the original free-flight simulation are employed.

The lift, the drag and the pitching moment due to the aerodynamic forces during the three freeflight flapping period in the free-flight simulation with the smaller abdominal mass are shown in Fig. 16, and the coordinates of the thorax in the simulation are also shown in Fig. 17. The functional forms of the lift, drag and pitching moment in this simulation are qualitatively similar to those in the original free-flight simulation, but quantitatively different from them. The lift in this simulation is smaller than that in the original free-flight simulation. It results in small descent of the thoracic COM in spite of the total mass smaller than that in the original free-flight simulation. The smaller drag in this simulation also results in less recession of the thoracic COM. The pitching moment during the downstroke $t \approx T / 4$ in this simulation is larger (negatively smaller) than that in the original free-flight simulation. The longitudinal torque due to the gravity acting on the abdomen and the reaction torque due to the motions of the abdomen are smaller than that in the original free-flight simulation. These variations result in the better periodicity of the thoracic pitching angle at the end of the first flap. However, the deviation of the pitching angle from the observed periodic trajectory gradually increases. It is of interest that the pitching angle at the end of the first flap is smaller than the initial pitching angle, but the butterfly is finally turned upward. It suggests that the deviation of the pitching angle from the observed trajectory does not increase monotonically. It must be noted that the trajectory of the pitching angle depends also on the phase of the flapping motion at which the extra control forces are removed.

Moreover, the trajectories of the pitching moment are sensitive to the mean approximate leadlag angle. The observed mean approximate lead-lag angle is $\bar{\eta} \approx-9.1 \times 10^{-2} \mathrm{rad}$. When the mean approximate lead-lag angle $2 \bar{\eta}$ is adopted, the thoracic pitching angle is approximately $0.1 \mathrm{rad}$ at the end of the first flap and the butterfly eventually falls forward. On the other hand, when the mean approximate lead-lag angle $7 \bar{\eta} / 4$, the butterfly makes a trajectory of the thoracic pitching angle 


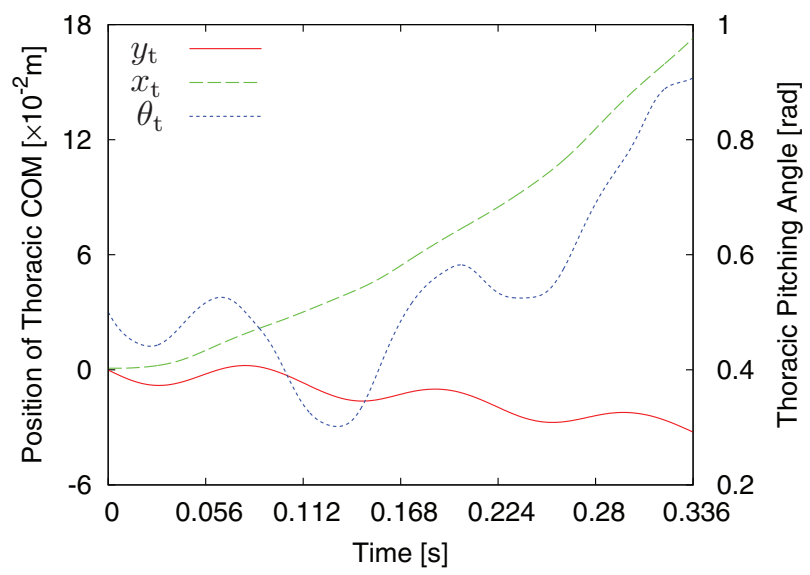

FIG. 17. Thoracic coordinates during the three free-flight flapping periods with the smaller abdominal mass $3 m_{\mathrm{a}} / 4$.

during three flapping periods similar to that in the free-flight simulation with the smaller abdominal mass. The slight difference of the flapping parameters causes the fatal difference in the thoracic pitching angle. It suggests the possibility of the control of the pitching angle by the flapping motion.

These behaviors of the pitching angle indicate that the trajectory in the phase space of the forward flight of the butterfly is an unstable periodic orbit. The forward flight of the butterfly is longitudinally unstable unlike most human-made airplanes designed to be longitudinally stable. The control of the thoracic pitching angle is essential to stabilize the flights. The control should be made dynamically by the flapping motion, and it would be mainly by the abdominal motion. The abdomen is presumed to be used as a rudder during the flights of tobacco hornworm driven by the ventrolongitudinal muscles of the metathorax, the dorsolongitudinal muscles of the first abdominal segment, and the dorsoventral muscles of the first abdominal segment. ${ }^{46}$ The chestnut tiger butterflies have similar thick muscle complex. Thus, it is deduced that the chestnut tiger butterflies explicitly control the abdominal joint. In contrast, the instability triggers complex trajectories characteristic to the flights of butterflies and may be the source of the strategy to avoid predation.

\section{v. CONCLUSION}

In this study, the flapping flights of the butterflies in the flow inflowing with constant velocities were numerically simulated. The butterfly is modeled as four rigid bodies, which are a spheroidal thorax, a spheroidal abdomen, and left and right thin flat wings. The wings as well as the abdomen are connected to the thorax with joints with three degrees of freedom. The interactions between the flow field and the butterfly are numerically constructed by the immersed boundary method. The three kinds of the numerical simulations, the tethered simulation, the prescribed simulation and the free-flight simulations, are made.

In the tethered simulation, the thorax of the butterfly is fixed, and the joint angles are adopted from the corresponding experiment. The forces to fix the thorax are compared with the forces measured with a force-torque sensor in the tethered experiment. The upward and streamwise forces in the simulation show a good agreement with the experimental observation. The reaction torques due to the flapping motion account for a large fraction of the longitudinal torque about the center of mass of the thorax. Both the simulation and the experiment require improvement of the accuracy with respect to the longitudinal torques. The aerodynamic lift is generated during downstrokes, and the aerodynamic thrust during upstrokes. The aerodynamic pitching moment about the center of mass of the thorax is negative during downstrokes, and is positive during upstrokes. The passive tracers in the wake in the simulation show zig-zag patterns similar to those in the experiment.

In the prescribed simulation, the thoracic coordinates as well as the joint angles are adopted from the free-flight experiment. The extra forces applied to the thorax, which the living butterflies cannot make directly, are required to realize the observed trajectories of the butterfly. Although the 
extra forces are as large as the aerodynamic forces, the powers due to the extra forces are much smaller than the powers due to the controllable joints. It suggests that the control to make steady periodic flights by dynamically changing the flapping motion is possible from the biological point of view. The characteristic vortical structures are found in the wakes and on the wings. The wing-tip vortices formed during downstrokes, which make multiplex helical structures, provide the lift and those during upstrokes provide the thrust. The trailing-edge vortices shed at the transitions from downstrokes to upstrokes also provide the lift. The leading-edge vortices separated on the leading edges during downstrokes are classified into two classes: one appears near the wing root and the other near the wing tip. The complex vortical structures similar to the flow behind the high angle-of-attack inclined plates are found on the wings below the leading-edge vortices near the wing root during the downstrokes. The secondary flow vortices below the leading-edge vortices are released to the wake between the downstrokes and the upstrokes, and the mechanism of the release is necessary for the steady periodic flapping flights.

In the free-flight simulation, where the joint motions are adopted from the free-flight experiment, the flapping motions can produce sufficient lift against the gravity. However, the butterfly in the simulation was turned upward and could not maintain the attitude in a proper range because the aerodynamic pitching moment was negatively smaller than the longitudinal torque due to the gravity acting on the abdomen. Then, the free-flight simulation failed to realize a steady periodic flight. On the other hand, in the free-flight simulations with smaller abdominal mass and with smaller mean approximate lead-lag angle, the flights were more stable than in the original free-flight simulation.

The pitching angle of the thorax is the key to the periodic flights. The periodic flapping flights of the butterfly are longitudinally unstable and the control of the pitching angle is essential. In this study, the flight stability was recovered by changing the abdominal mass or the mean approximate lead-lag angle. Obviously, however, the living butterflies cannot change the abdominal mass easily. In addition, the static change of the flapping parameters cannot realize a perfect periodic flight although it makes the periodicity better. In fact, the living butterflies should dynamically control their attitude by sensing their instantaneous attitude. Moreover, the living butterflies redundantly have a large number of degrees of freedom in the flapping motions to explicitly stabilize the flights. The explicit control is considered to be made mainly with the abdominal motions. The flexibility such as the elastic deformation of the wings are also anticipated to stabilize the flapping flights and to function as an implicit control. It is our future work to identify the mechanism of the explicit and implicit controls.

\section{ACKNOWLEDGMENTS}

Numerical computation in this work was carried out at the Yukawa Institute Computer Facility. This work was partially supported by Grants-in-Aid for Scientific Research, MEXT.

\section{APPENDIX: VERIFICATION OF NUMERICAL PARAMETERS}

The computational parameters in this paper are verified in this Appendix. The flapping parameters which are the same in the tethered simulation in Sec. III A are adopted. The computational domain is a cube $L$ on a side in all the numerical tests in this Appendix. The reference parameters denoted by Test 1 are the same parameters as the tethered simulation except for the computational domain length $L=0.25 \mathrm{~m}$, the numbers of the grid points $N_{x}=2048$, and $N_{y}=N_{z}=512$. The parameters denoted by Test 2 are the same as in Test 1 except $v=4 v_{\text {air }}$. Test 3 is made in a larger computational cube whose side is $L=0.375 \mathrm{~m}$. Note that the numbers of the grid points are changed to $N_{x}=3072$, and $N_{y}=N_{z}=768$ in Test 3 so that the grid spacings are the same as those in Test 1. The time increment $\Delta t=T / 5000=2.4 \times 10^{-5} \mathrm{~s}$ in Test 4 is smaller than $\Delta t=T / 4000=3$ $\times 10^{-5} \mathrm{~s}$ in Test 1 . The upward and streamwise forces and the longitudinal torque in the four tests are shown in Fig. 18.

The values of the kinematic viscosity twice or thrice larger than that of the actual kinematic viscosity of the air are adopted in this paper. It is validated by comparison between Test 1 and Test 2. The difference of the forces and torque between them is little, although the fine vortical structures 


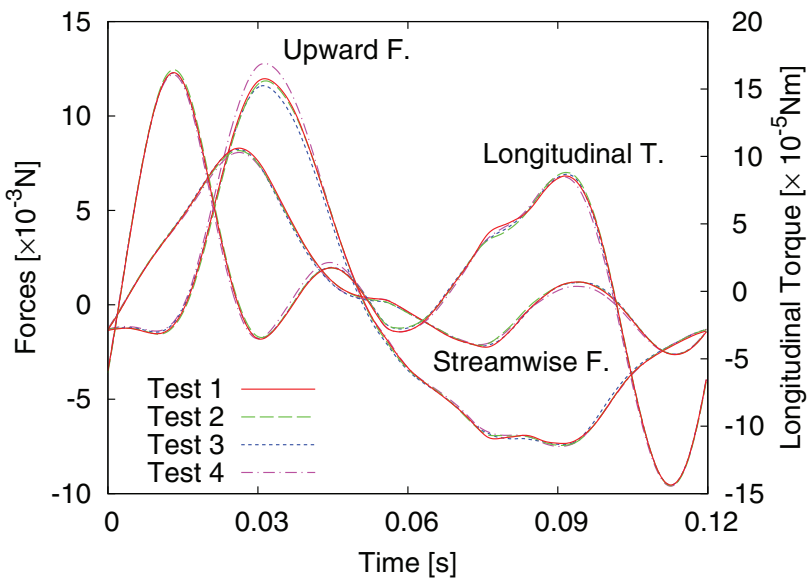

FIG. 18. Upward and streamwise forces and longitudinal torque in four tests for computational validation.

are less visible in Test 2 . The dependence of the forces and the torque on the viscosity is practically negligible.

The streamwise length of the computational domain and the periodic boundary conditions of the lateral $y$ and $z$ directions are validated by comparison between Test 1 and Test 3 . The small difference appears in the middle of the downstroke around $t=T / 4$. The maximal upward force in Test 3 is $4 \times 10^{-4} \mathrm{~N}$ smaller than that in Test 1 . The influence of time increment is also compared between Test 1 and Test 4 . The maximal upward force in Test 4 is $8 \times 10^{-4} \mathrm{~N}$ larger than that in Test 1. On the whole, the influences of the computational domain and of the time increment are small enough, although the differences are slightly seen in the middle of the downstroke.

${ }^{1}$ S. P. Sane, "The aerodynamics of insect flight," J. Exp. Biol. 206, 4191-4208 (2003).

${ }^{2}$ Z. J. Wang, "Dissecting insect flight," Annu. Rev. Fluid Mech. 37, 183-210 (2005).

${ }^{3}$ F.-O. Lehmann, "The mechanisms of lift enhancement in insect flight," Naturwiss. 91, 101-122 (2004)

${ }^{4}$ M. Iima and T. Yanagita, "Is a two-dimensional butterfly able to fly by symmetric flapping?" J. Phys. Soc. Jpn. 70, 5-8 (2001).

${ }^{5} \mathrm{~N}$. Vandenberghe, J. Zhang, and S. Childress, "Symmetry breaking leads to forward flapping flight," J. Fluid Mech. 506, 147-155 (2004).

${ }^{6}$ A. J. Bergou, L. Ristroph, J. Guckenheimer, I. Cohen, and Z. J. Wang, "Fruit flies modulate passive wing pitching to generate in-flight turns," Phys. Rev. Lett. 104, 148101 (2010).

${ }^{7}$ D. E. Alexander and S. Vogel, Nature's Flyers: Birds, Insects, and the Biomechanics of Flight (Johns Hopkins University Press, Baltimore, 2004).

${ }^{8}$ D. Altshuler, M. Princevac, H. Pan, and J. Lozano, "Wake patterns of the wings and tail of hovering hummingbirds," Exp. Fluids 46, 835-846 (2009).

${ }^{9}$ L. C. Johansson, M. Wolf, R. von Busse, Y. Winter, G. R. Spedding, and A. Hedenström, "The near and far wake of Pallas' long tongued bat (Glossophaga soricina)," J. Exp. Biol. 211, 2909-2918 (2008).

${ }^{10}$ H. Dong, R. Mittal, and F. M. Najjar, "Wake topology and hydrodynamic performance of low-aspect-ratio flapping foils," J. Fluid Mech. 566, 309-343 (2006)

${ }^{11}$ A. K. Brodsky, "Vortex formation in the tethered flight of the peacock butterfly Inachis io L. (Lepidoptera, Nymphalidae) and some aspects of insect flight evolution," J. Exp. Biol. 161, 77-95 (1991).

${ }^{12} \mathrm{H}$. Aono, F. Liang, and H. Liu, "Near- and far-field aerodynamics in insect hovering flight: An integrated computational study," J. Exp. Biol. 211, 239-257 (2008).

${ }^{13}$ R. Ramamurti and W. C. Sandberg, "A three-dimensional computational study of the aerodynamic mechanisms of insect flight,” J. Exp. Biol. 205, 1507-1518 (2002).

${ }^{14}$ M. H. Dickinson and K. G. Götz, "The wake dynamics and flight forces of the fruit fly Drosophila melanogaster," J. Exp. Biol. 199, 2085-2104 (1996).

${ }^{15}$ C. P. Ellington, C. van den Berg, A. P. Willmott, and A. L. R. Thomas, "Leading-edge vortices in insect flight," Nature (London) 384, 626-630 (1996).

${ }^{16}$ R. B. Srygley and A. L. R. Thomas, "Unconventional lift-generating mechanisms in free-flying butterflies," Nature (London) 420, 660-664 (2002).

${ }^{17} \mathrm{X}$. X. Wang and Z. N. Wu, "Stroke-averaged lift forces due to vortex rings and their mutual interactions for a flapping flight model," J. Fluid Mech. 654, 453-472 (2010).

${ }^{18}$ J. H. Wu and M. Sun, "Unsteady aerodynamic forces of a flapping wing," J. Exp. Biol. 207, 1137-1150 (2004). 
${ }^{19}$ H. Nagai, K. Isogai, T. Fujimoto, and T. Hayase, "Experimental and numerical study of forward flight aerodynamics of insect flapping wing," AIAA J. 47, 730-742 (2009).

${ }^{20}$ B. Cheng, S. N. Fry, Q. Huang, and X. Deng, "Aerodynamic damping during rapid flight maneuvers in the fruit fly Drosophila,” J. Exp. Biol. 213, 602-612 (2010).

${ }^{21}$ A. Azuma, The Biokinetics of Flying and Swimming (AIAA, Virginia, 2006).

${ }^{22}$ U. Pesavento and Z. J. Wang, "Flapping wing flight can save aerodynamic power compared to steady flight," Phys. Rev. Lett. 103, 118102 (2009).

${ }^{23}$ Z. J. Wang, "Vortex shedding and frequency selection in flapping flight," J. Fluid Mech. 410, 323-341 (2000).

${ }^{24}$ Q. Zhu, "Optimal frequency for flow energy harvesting of a flapping foil," J. Fluid Mech. 675, 495-517 (2011).

${ }^{25}$ S. P. Sane, A. Dieudonné, M. A. Willis, and T. L. Daniel, "Antennal mechanosensors mediate flight control in moths," Science 315, 863 (2007).

${ }^{26}$ M. Srinivasan, S. Zhang, M. Lehrer, and T. Collett, "Honeybee navigation en route to the goal: visual flight control and odometry,” J. Exp. Biol. 199, 237-244 (1996).

${ }^{27}$ R. Dudley, The Biomechanics of Insect Flight: Form, Function, Evolution (Princeton University Press, Princeton, 2002).

${ }^{28}$ T. L. Hedrick and T. L. Daniel, "Flight control in the hawkmoth Manduca sexta: The inverse problem of hovering," J. Exp. Biol. 209, 3114-3130 (2006).

${ }^{29}$ X. Deng, L. Schenato, and S. S. Sastry, "Flapping flight for biomimetic robotic insects: Part II—flight control design," IEEE Trans. Rob. 22, 789-803 (2006).

${ }^{30} \mathrm{H}$. Tanaka and I. Shimoyama, "Forward flight of swallowtail butterfly with simple flapping motion," Bioinsp. Biomim. 5, 026003 (2010).

${ }^{31}$ R. Mittal and G. Iaccarino, "Immersed boundary methods," Annu. Rev. Fluid Mech. 37, 239-261 (2005).

${ }^{32}$ X. Yang, X. Zhang, Z. Li, and G.-W. He, "A smoothing technique for discrete delta functions with application to immersed boundary method in moving boundary simulations," J. Comput. Phys. 228, 7821-7836 (2009).

${ }^{33}$ K. Senda, N. Yokoyama, K. Yokoi, and M. Kitamura, "Modeling for structural flexibility of wings in flapping butterfly," in Computational Intelligence and Bioinformatics/755: Modelling, Identification, and Simulation, edited by T. Znati, Z. Bar-Joseph, and L. Rothrock (ACTA Press, Calgary, 2011).

${ }^{34}$ The flapping motion and the lead-lag motion are normally defined as the wing motions normal to and parallel to the long axis of the thorax, respectively. The feathering motion is also defined as the variation of the pitching angle of the wing. Thus, the often-used flapping, lead-lag, and feathering angles are ill-defined to construct the three-dimensional motion of the wing.

${ }^{35}$ K. Senda, T. Obara, M. Kitamura, T. Nishikata, N. Hirai, M. Iima, and N. Yokoyama, "Modeling and emergence of flapping flight of butterfly based on experimental measurements," Robot. Auton. Syst. 60, 670-678 (2012).

${ }^{36}$ K. Senda, T. Obara, M. Kitamura, N. Yokoyama, N. Hirai, and M. Iima, "Effects of structural flexibility of wings in flapping flight of butterfly," Bioinspir. Biomim. 7, 025002 (2012).

${ }^{37} \mathrm{~K}$. Suzuki and T. Inamuro, "Effect of internal mass in the simulation of a moving body by the immersed boundary method," Comput. Fluids 49, 173-187 (2011).

${ }^{38}$ P. R. Ehrlich and S. E. Davidson, "The internal anatomy of the monarch butterfly, Danaus plexippus L.," Microentomology 24, 85-133 (1961).

${ }^{39}$ J. C. R. Hunt, A. Wray, and P. Moin, "Eddies, stream, and convergence zones in turbulent flows," Center for Turbulence Research Report CTR-S88 (1988), p. 193.

${ }^{40}$ See supplementary material at http://dx.doi.org/10.1063/1.4790882 for movies.

${ }^{41}$ H. Liu and K. Kawachi, “A numerical study of insect flight,” J. Comput. Phys. 146, 124-156 (1998).

${ }^{42}$ M. G. Hall, "Vortex breakdown," Annu. Rev. Fluid Mech. 4, 195-218 (1972).

${ }^{43}$ J. Young, S. M. Walker, R. J. Bomphrey, G. K. Taylor, and A. L. R. Thomas, "Details of insect wing design and deformation enhance aerodynamic function and flight efficiency," Science 325, 1549-1552 (2009).

${ }^{44}$ T. Nakata and H. Liu, "Aerodynamic performance of a hovering hawkmoth with flexible wings: A computational approach," Proc. R. Soc. London, Ser. B 279, 722-731 (2012).

${ }^{45} \mathrm{Y}$. Hu and J. J. Wang, "Dual leading-edge vortex structure for flow over a simplified butterfly model," Exp. Fluids 50, 1285-1292 (2011).

${ }^{46}$ J. L. Eaton, Lepidopteran Anatomy (Wiley, New York, 1988). 